\title{
Revisiting the Cygnus OB associations
}

\author{
Alexis L. Quintana ${ }^{\circledR \star}$ and Nicholas J. Wright ${ }^{\circledR}$ \\ Astrophysics Group, Keele University, Keele ST5 5BG, UK
}

Accepted 2021 September 13. Received 2021 September 13; in original form 2021 August 5

\begin{abstract}
OB associations play an important role in Galactic evolution, though their origins and dynamics remain poorly studied, with only a small number of systems analysed in detail. In this paper, we revisit the existence and membership of the Cygnus OB associations. We find that of the historical OB associations only Cyg OB2 and OB3 stand out as real groups. We search for new OB stars using a combination of photometry, astrometry, evolutionary models, and an SED-fitting process, identifying 4680 probable OB stars with a reliability of $>90$ per cent. From this sample, we search for OB associations using a new and flexible clustering technique, identifying six new OB associations. Two of these are similar to the associations Cyg OB2 and OB3, though the others bear no relationship to any existing systems. We characterize the properties of the new associations, including their velocity dispersions and total stellar masses, all of which are consistent with typical values for OB associations. We search for evidence of expansion and find that all are expanding, albeit anisotropically, with stronger and more significant expansion in the direction of Galactic longitude. We also identify two large-scale $\left(160 \mathrm{pc}\right.$ and $\left.25 \mathrm{~km} \mathrm{~s}^{-1}\right)$ kinematic expansion patterns across the Cygnus region, each including three of our new associations, and attribute this to the effects of feedback from a previous generation of stars. This work highlights the need to revisit the existence and membership of the historical OB associations, if they are to be used to study their properties and dynamics.
\end{abstract}

Key words: stars: distances - stars: early-type - stars: kinematics and dynamics - stars: massive-open clusters and associations: individual: Cyg OB1, Cyg OB2, Cyg OB3, Cyg OB8, Cyg OB9.

\section{INTRODUCTION}

Many OB stars are assembled in associations (McKee \& Williams 1997). They were first defined by Ambartsumian (1947) as gravitationnally unbound groups of young stars with a high concentration of OB stars. They typically have total masses ranging from a few thousands to a few tens of thousands of stellar masses, with a density lower than $0.1 \mathrm{M}_{\odot} \mathrm{pc}^{-3}$ and a young age (necessary to explain their kinematic and spatial concentration despite their unbound nature). They often extend over tens of parsecs and include smaller groups of different ages and kinematics (Garmany 1994; Wright 2020). They have a huge effect on their environment: The feedback from their massive members creates $\mathrm{H}$ II regions, superbubbles and dissociation in the diffuse interstellar medium (Dove \& Shull 1994; Shull \& Saken 1995). As a transitional phase between star-forming regions and the field population of stars, OB associations are important to study in order to better understand Galactic evolution (Wright 2020).

The two main scenarios for the origin of $\mathrm{OB}$ associations are the expanding star cluster and hierarchical star formation models. According to the former, $\mathrm{OB}$ associations constitute the expanded remnants of dense star clusters (Blaauw 1964), following a disruption of their parent molecular cloud through a feedback phase once massive stars emerge (Hills 1980). According to the latter, stars form in groups with a wide variety of sizes and densities and most are unbound and disperse from birth (Kruijssen 2012). It is therefore considered important to search for expansion patterns in

^E-mail: a.l.p.quintana.isasi@keele.ac.uk
OB associations. This has been possible over the last few years thanks to data from various astrometric surveys, predominantly from the Gaia mission (Gaia Collaboration 2016). The results so far have been mixed, with Wright et al. (2016), Wright \& Mamajek (2018), and Ward \& Kruijssen (2018) failing to detect expansion, while Melnik \& Dambis (2020) measured an expansion signature in only 6 of the $28 \mathrm{OB}$ associations they studied. On the other hand, more recent studies that have used a revised kinematic selection of the members of OB associations have found expansion in several associations and their subgroups (Kounkel et al. 2018, Cantat-Gaudin et al. 2019, Armstrong et al. 2020). Such findings encourage a deeper investigation.

The goal of this work is to study the kinematics of OB associations to search for evidence of expansion, using reliable and updated membership lists. This requires us to either refine the membership of existing OB associations or identify such systems from scratch. To that aim, we turn our attention to the Cygnus region as a first target since it contains many OB associations and is rich in OB stars (Reipurth \& Schneider 2008).

The Cygnus region of the Galactic plane is home to a considerable amount of recent star formation, including the massive Cygnus $\mathrm{X}$ star-forming region, thousands of OB stars, H II regions, supernova remnants and nine OB associations (Mel'Nik \& Efremov 1995; Reipurth \& Schneider 2008). Since the study of Massey \& Thompson (1991), the highly reddened OB star population of Cygnus OB2 has been studied by many authors, most recently by Wright, Drew \& Mohr-Smith (2015) and Berlanas et al. (2020). The association is home to approximately 70 O-type stars with ages between 1 and $7 \mathrm{Myr}$ and a total mass of $1.65 \times 10^{4} \mathrm{M}_{\odot}$ (Wright et al. 2015). 
However, Berlanas et al. (2019) recently showed that the association appears to constitute two structures superimposed along the line of sight: the main association at a distance of $\sim 1.76 \mathrm{kpc}$ and a smaller, foreground population at $\sim 1.35 \mathrm{kpc}$. Studies of the kinematics of Cyg OB2 have to date failed to identify a clear expansion signature (Wright et al. 2016; Arnold, Goodwin \& Wright 2020). The other OB associations in Cygnus remain poorly studied despite the available information (see e.g. Reipurth \& Schneider 2008; Comerón \& Pasquali 2012; Mel'nik \& Dambis 2017; Melnik \& Dambis 2020) and deserve as a consequence a better understanding.

In this paper, we investigate the five main $\mathrm{OB}$ associations in Cygnus, consider their reality as genuine associations, and eventually propose the existence of six alternative structures in the region, some of which overlap with the historical associations. In Section 2, we analyse the historical $\mathrm{OB}$ associations and show that, apart from Cyg OB2 and OB3, they do not stand out as real groups. In Section 3, we describe how we identify OB stars across the region using photometry, astrometry, evolutionary models, and a new SED-fitting tool. In Section 4, we use a new flexible clustering technique to identify new associations. In Section 5, we characterize the identified groups by studying their physical and kinematic properties. In Section 6, we discuss these findings and in Section 7, we wrap up our results.

\section{THE EXISTING OB ASSOCIATIONS IN CYGNUS}

In this section, we consider the stellar content and reality of the existing $\mathrm{OB}$ associations in Cygnus using modern photometry and astrometry.

Our focus is on a $60 \mathrm{deg}^{2}$ area in the Cygnus constellation spanned by $1=\left[71^{\circ}, 81^{\circ}\right]$ and $\mathrm{b}=\left[-1^{\circ}, 5^{\circ}\right]$. This region contains the $\mathrm{OB}$ associations Cyg OB1, OB2, OB3, OB8, and OB9, the five most well-studied associations in Cygnus. A total of 178 OB stars were identified by Blaha \& Humphreys (1989) and divided between these five associations based only on their positions on the sky. The other OB associations in Cygnus, i.e. Cyg OB4, OB5, OB6, and OB7 are not considered here because they are much more extended and found at greater longitudes. For this work, we use the list of 164 OB stars in Cyg OB1, OB3, OB8, and OB9 from Blaha \& Humphreys (1989). For Cyg OB2, we use the updated census of OB stars from Berlanas et al. (2019). This gave us a total of 341 OB stars.

These sources were cross-matched with Gaia EDR3 data using a matching radius of 1 arcsec, with 329 sources matched successfully. These data were filtered by applying the recommended astrometric criterion from Lindegren et al. (2020b), that their renormalized unit weight error (RUWE) should be lower than 1.4. 269 sources fulfilled these conditions. Distances of sources with good astrometry were obtained from Bailer-Jones et al. (2020).

Fig. 1 shows the distribution of these sources in both Galactic coordinates (where all stars are shown) and with distance plotted against $l$ (where only sources that passed the astrometric quality tests are shown). Most associations are at similar distances, between 1.5 and $2.0 \mathrm{kpc}$, with a slight trend of increasing distance with decreasing Galactic longitude. Cyg OB2 is the most distinct and highly concentrated of the associations, with the other associations less concentrated, though all associations appear to include objects whose distances or kinematics (Fig. 2) would imply they are unlikely to be part of these associations.

We attempted to refine the association membership, as originally defined by Blaha \& Humphreys (1989), by removing outliers in distance and proper motion, and also used a number of modern clustering algorithms applied to this list of OB stars to 're-discover' these associations, but only Cyg OB2 and OB3 appeared to show any level of kinematic coherence that would suggest they are true associations. We can only conclude that the other associations, Cyg OB1, OB8, and OB9, are not genuine OB associations. It would therefore be pertinent to reconsider the possible $\mathrm{OB}$ associations in Cygnus, starting from the ground up.

\section{IDENTIFYING AND CHARACTERIZING OB STARS}

In this section, we outline our photometric SED-fitting technique used to identify OB stars across our region of study and estimate their physical parameters.

\subsection{Data and selection process}

We use photometry and astrometry from IGAPS (INT Galactic Plane Survey, providing $g_{-}, r$-, $i$-band photometry, Drew et al. 2005, Monguió et al. 2020), 2MASS (Two-Micron All-Sky Survey, providing $J-, H-$, and $K_{\mathrm{s}}$-band photometry, Cutri et al. 2003), UKIDSS (United Kingdom Infrared Deep Sky Survey, providing deeper $J$-, $H$ - and $K$-band photometry, Lucas et al. 2008), and Gaia EDR3 (Gaia Collaboration 2016, 2020; Riello et al. 2020), providing $G-, G_{\mathrm{BP}}-$ and $G_{\mathrm{RP}}$-band photometry. In addition, we use parallaxes and proper motions from Gaia EDR3 (Fabricius et al. 2021), and the probabilistic distances derived from these parallaxes provided by Bailer-Jones et al. (2020). We correct all parallaxes for the nonzero parallax zero-point, whose value has been shown to depend on magnitude, ecliptic latitude, and the pseudocolour of the source, using the prescription in Lindegren et al. (2020a).

There are a total of 8474415 sources in Gaia EDR3 in this region of study, 7434799 of which have a valid five or six-parameter astrometric solution (suitable for our needs). We cross-match this sample with 2MASS, UKIDSS, and IGAPS, using a matching radius of 1 arcsec, using the proper motions to account for the epoch difference. We found near-IR matches for 6347730 sources and IG APS matches for 6158261 sources. We discarded the 1087069 Gaia sources that lacked a near-IR counterpart as a near-IR photometric data point is necessary for our SED fitting. These sources generally have $G>18$, too faint to be detected by 2MASS and lying in one of the areas of incomplete coverage for UKIDSS. They are unlikely to correspond to $\mathrm{OB}$ stars in our region of interest.

We discarded all unreliable photometry. For IGAPS, photometry is reliable if saturation is avoided and if its associated class indicates a star or probable star (Monguió et al. 2020). For Gaia, the requirement summarizes as $\left|C^{*}\right|<3 \sigma_{C *}$ where $C^{*}$ stands for the corrected excess flux factor in the $G_{\mathrm{RP}}$ and $G_{\mathrm{BP}}$ bands and $\sigma_{C *}$ is a power law on the $G$ band with a chosen $3 \sigma$ level (Riello et al. 2020). For 2MASS, a goodquality flag on each photometric band is required (Cutri et al. 2003). For UKIDSS, we impose ErrBits $<256$, otherwise this indicated a bad value. We exclude as well photometry with either $J<13.25, H$ $<12.75$, and $K<12$, below which the photometry risks saturation (Lucas et al. 2008).

Finally, we require at least one good photometric measurement in a blue band ( $g, B P$ or $G$ ) and one in a near-IR band, to ensure reliable SED fits, which reduces the size of our sample to 5399862 sources.

To ensure the quality of the astrometry, we require $R U W E<1.4$, leaving 5211036 sources. We go further by imposing $\left|\frac{\sigma}{q * \sigma_{\sigma}}\right|>2$, where $\sigma_{\varpi}$ is the standard parallax uncertainty, and $q$ is a multiplicative factor that inflates the published standard uncertainty on parallaxes as it was found to be underestimated, notably for 

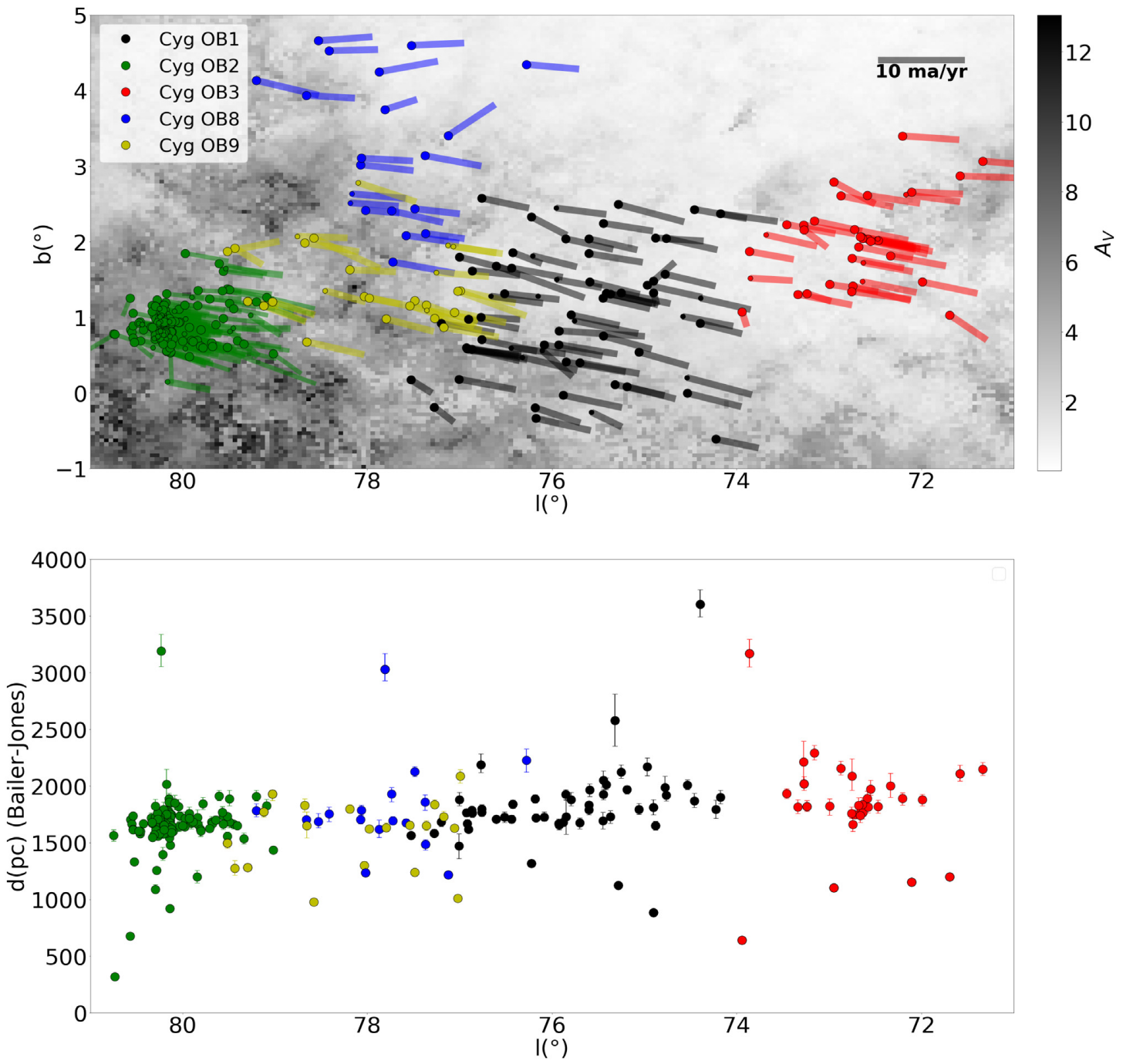

Figure 1. Top panel: spatial distribution of Cygnus OB association members from Blaha \& Humphreys (1989) and Berlanas et al. (2019), displayed with their proper motion as a vector, with a background extinction map from Green et al. (2019), representing the integrated reddening up to a distance of $1.8 \mathrm{kpc}$. Objects with $R U W E>1.4$ have been displayed as small circles (whose proper motions may be questionable). Bottom panel: distance as a function of Galactic longitude for sources with $R U W E<1.4$, with distances and uncertainties taken from Bailer-Jones et al. (2020).

bright sources (El-Badry, Rix \& Heintz 2021). This leaves 2145918 sources. ${ }^{1}$

We showed in Section 2 that most of the stars in the Cygnus OB associations are located between 1 and $2.5 \mathrm{kpc}$. Therefore, we removed all sources that fall outside this range of distances using the geometric values calculated by Bailer-Jones et al. (2020), trimming the sample to 896946 sources.

Since we are interested primarily in OB stars, we limit our sample to sources with absolute magnitudes, $M_{\mathrm{K}}<1.07$, where $M_{\mathrm{K}}=K-$ $5 \log (d)+5$ with $d$ standing for the distance from Bailer-Jones et al. (2020), as the $K$ band is the least affected by extinction. This value

\footnotetext{
${ }^{1}$ Maíz Apellániz, Pantaleoni González \& Barbá (2021) offer an alternative method for filtering Gaia data and correcting the astrometry that involves relaxing the constraint on RUWE and inflating the parallax uncertainties. We found that this approach typically increased the parallax errors by 10 20 per cent, but would have made our final sample 5 per cent larger (as the RUWE constraint is slightly relaxed). This change would therefore not have brought a significant change to our results.
}

corresponds to spectral types earlier than A0V (Pecaut \& Mamajek 2013). However, for sources that lack $K$-band photometry, we use $M_{\mathrm{H}}$ $<1.10$ or $M_{\mathrm{J}}<1.07$, which offer equivalent criteria in the $J$ and $H$ bands. With these conditions, the sample is reduced to 47498 sources.

Finally, we use the near-IR colour-colour diagram to remove giants by retaining only objects that have larger $H-K$ colours than the dashed line in Fig. 3 (projecting UKIDSS photometry into the 2MASS system using the equations from Hodgkin et al. 2009). This line separates early-type stars of any reddening from the majority of red giants. Sources that lacked sufficient photometry to be plotted in this diagram were retained regardless.

Following all cuts, we were left with a sample of 20498 objects that will be further classified using SED fitting. From the 152 sources listed by Blaha \& Humphreys (1989), 87 passed all these tests.

\subsection{SED Fitting}

In this section, we describe our SED-fitting process that is used to estimate the physical properties of our targets and subsequently select the OB stars we are interested in. We fit the observed SEDs and 


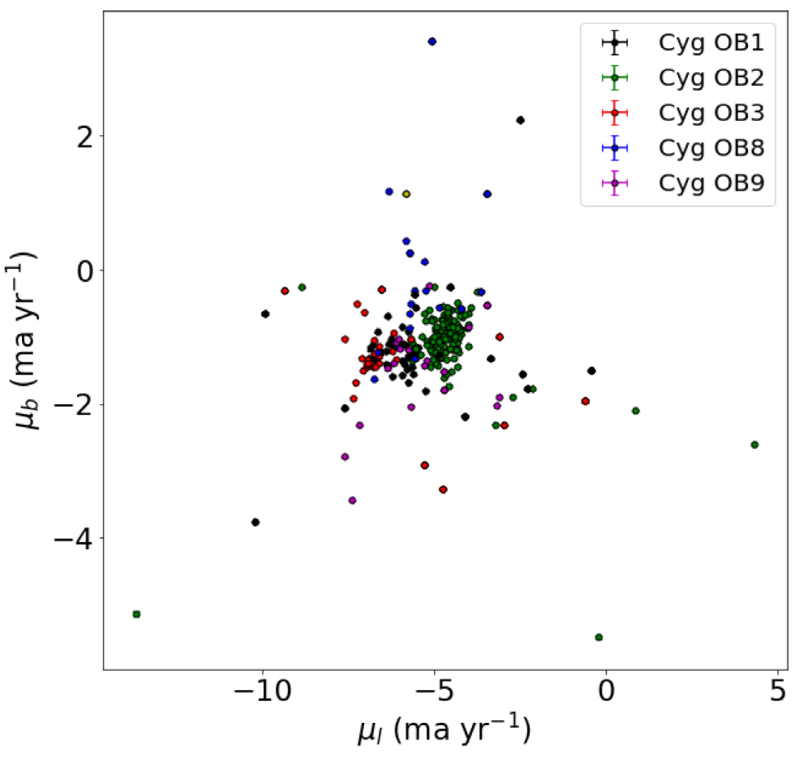

Figure 2. Galactic proper motion distribution for the 121 objects from Blaha \& Humphreys (1989) and 148 objects from Berlanas et al. (2019) that passed the astrometric quality cut, with error bars shown (the majority of which are smaller than the plotted symbol). It is clear that, with the exception of $\mathrm{Cyg} \mathrm{OB} 2$ and $\mathrm{OB} 3$, the other associations are not kinematically distinct or coherent.

parallaxes to a series of model SEDs using a Markov chain Monte Carlo (MCMC) process to explore the parameter space and constrain the posterior distribution. Here, we describe the models used and the fitting process adopted.

\subsubsection{Model SEDs}

Stellar SEDs can generally be parameterized by the combination of effective temperature, $T_{\text {eff }}$, luminosity, $L$, distance, $d$, and extinction, $A_{V}$. In some situations, it can also be useful to parameterize the form of the reddening law used, for example, using $R_{V}$. Main-sequence models can be simplified by relying on a simple relationship (or model-predicted dependency) between $T_{\text {eff }}$ and $L$, though this can lead to uncertainties if the population to be fitted includes post-mainsequence stars such as luminous red giants.

Instead of using $T_{\text {eff }}$ and $L$ to parameterize the unreddened SED, we utilize stellar evolutionary models that predict these parameters as a function of initial mass, $M$, and age. Furthermore, rather than using both distance and extinction as free parameters, we exploit the availability of 3D extinction maps, in this case, the one produced by Green et al. (2019), to remove extinction as a free parameter and instead derive it from the distance. ${ }^{2}$ Finally, we removed $R_{V}$ as a free parameter as studies in this area have shown that it does not vary significantly from the mean Galactic value of $R_{V}=3.1$ (Wright et al. 2015).

We calculate our model SEDs using spectroscopy from two stellar spectral libraries, the Kurucz synthetic stellar library (Coelho 2014), for models with $T_{\text {eff }}$ between 4000 and $20000 \mathrm{~K}$, and the Tubingen NLTE Model Atmosphere Package (Werner \& Dreizler 1999; Rauch \& Deetjen 2003; Werner et al. 2003), for models from 20000 to

\footnotetext{
${ }^{2}$ In early tests, we found that using both $A_{V}$ and distance as free parameters lead to a systematic underestimation of $A_{V}$ compared to estimates from 3D extinction maps and that by requiring this dependency of one parameter on the other we overcame this.
}

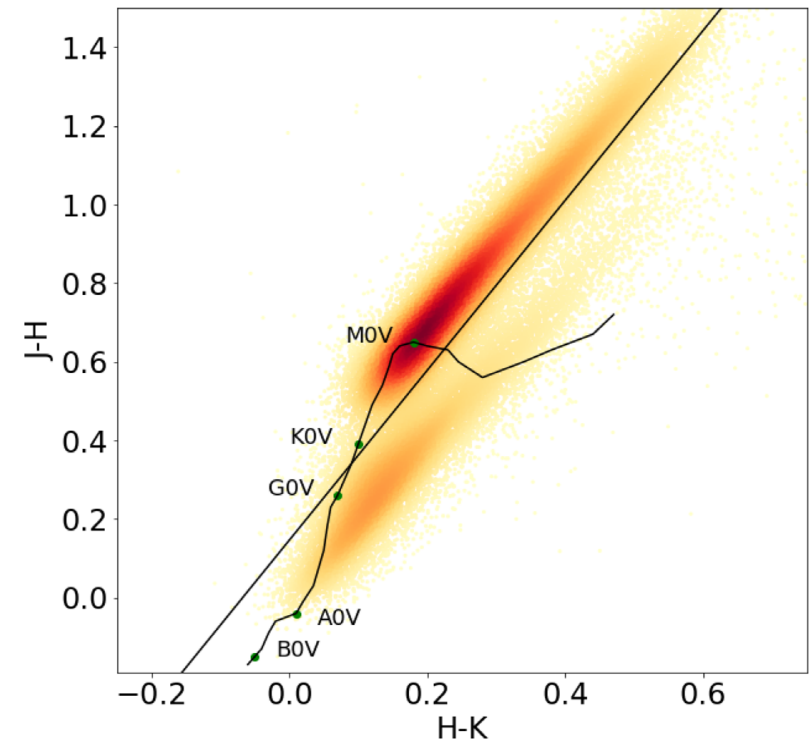

Figure 3. Near-IR colour-colour diagram displaying the source density from our photometric and astrometric sample. The black curve corresponds to the unreddened main-sequence from Straižys \& Lazauskaitė (2009). The black line, which is parallel to the near-IR reddening vector, is used to separate earlytype stars of any reddening (below the line) from red giants (above the line).

$50000 \mathrm{~K}$. In each case, we chose models at $\log (g)=4$ (except for the model at $T_{\text {eff }}=4000 \mathrm{~K}$ where we are forced to use $\log (g)=$ 4.5 instead), both using solar abundances. We will comment on the consequences of this choice in Section 3.2.4. The model spectra are reddened using the Fitzpatrick et al. (2019) reddening model with $R_{V}$ set to 3.1, a value consistent with the region (Wright et al. 2015).

The reddened model spectra are then convolved with the filter profiles introduced in Section 3.1, to derive synthetic magnitudes (Smith 2016):

$m_{x}=-2.5 \log \left[\frac{\int \lambda F_{\text {red }} T_{x} \mathrm{~d} \lambda}{\int \lambda F_{V} T_{x} \mathrm{~d} \lambda}\right]$,

where $T_{x}$ is the transmission curve of filter $\mathrm{x}$ and $F_{V}$ is a model Vega spectrum from Bohlin (2007).

To calibrate our model, we calculated synthetic colours for main-sequence stars and compared them to the empirical main sequence from Pecaut \& Mamajek (2013) for Gaia and 2MASS and Verbeek et al. (2012) for IGAPS. We found generally excellent agreement: the root mean squares differences for the colours $(g-$ $r, B P-R P, G-R P, J-H, H-K)$ were equal to $(0.025,0.012$, $0.037,0.005)$. The only band for which a discrepancy was noted was the $H$ band, for which we needed to add 0.0375 to all magnitudes to bring them in line with empirical values. We attribute this to either uncertainties in the modelled $\mathrm{OH}$ lines in stellar spectra or the $H$-band filter transmission function.

\subsubsection{Fitting process}

The list of model parameters, we are seeking to estimate are

$\theta=[\log ($ Mass $), \operatorname{Fr}($ Age $), d, \ln (f)]$,

where $\operatorname{Fr}(A g e)$ stands for the fractional age and $\ln (f)$ represents the logarithm of an additional uncertainty that needs to be considered to achieve agreement between the observations and the model. Adding such a parameter helps $\chi^{2}$ to converge to 1 for the computation of 

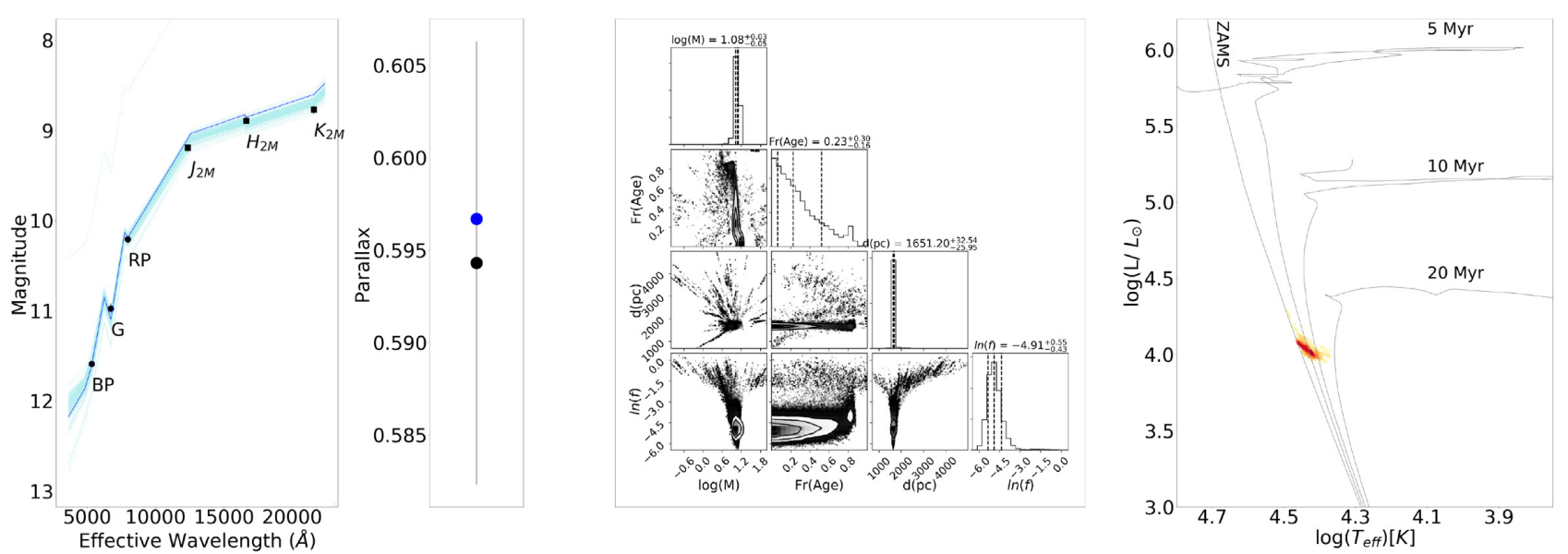

Figure 4. Results of the fit for J203227.75+41285.2 (Schulte 21), fitted with $\log \left(T_{\text {eff }}\right)=4.44 \pm 0.02$, compared with a spectroscopic $\log \left(T_{\text {eff }}\right)=4.45_{-0.05}^{+0.03}$ (Kiminki et al. 2007). Left-hand panel: SED fit with the data shown in black and inflated error bars. The blue line shows the best-fitting model whilst the cyan lines correspond to 100 randomly selected iterations from the posterior distribution. Centre left-hand panel: fitted parallax in blue versus Gaia parallax in black. Centre right-hand panel: Triangle plot showing the posterior distributions of the fitted parameters. Right-hand panel: posterior distributions of $\log \left(T_{\text {eff }}\right)$ and $\log \left(L / L_{\odot}\right)$ shown as a heat map in an HR diagram with a zero-age main-sequence and isochrones from Ekström et al. (2012).

the likelihood (Foreman-Mackey et al. 2013). The new errors will therefore be expressed as (e.g. Casey 2016)

$s^{2}=\sigma^{2}+f^{2}$ Model $^{2}$,

where Model is the combination of the model SED and the model parallax.

We define the following priors for these parameters:

$\ln (P(\theta))=\left\{\begin{array}{l}\log \left(\frac{1}{2 L^{3}} \mathrm{~d}^{2} \exp \left(\frac{-\mathrm{d}}{L}\right)\right) \text { if }\left\{\begin{array}{l}-1.0 \leq \log (\text { Mass }) \leq 2.0 \\ 0.0 \leq F r(\text { Age }) \leq 1.0 \\ 0.0 \leq d \leq 5000.0 \mathrm{pc} \\ -10.0 \leq \ln (f) \leq 1.0\end{array}\right. \\ -\infty \quad \text { otherwise }\end{array}\right.$

The prior on distance originates from Bailer-Jones (2015), and is designed to achieve an exponentially decreasing volume density of stars, using a scale length of $L=1.35 \mathrm{kpc}$.

The parameters of the model SED are constrained by comparing them to the observed SED using Bayesian inference and a maximumlikelihood test. Through this method, with a set of empirical data and model parameters, we can compute the posterior probability, labelled $P(\theta \mid d)$, which is the probability of a set of model parameters, given the observations, by applying Bayes' theorem:

$P(\theta \mid \mathrm{d}) \propto P(\mathrm{~d} \mid \theta) P(\theta)$,

where $P(\theta)$ are the priors and $P(\mathrm{~d} \mid \theta)$, named the likelihood, corresponds to the probability that the data are measured given the model parameters.

The observational data, consisting of the photometry and astrometry, and their uncertainties, can be written as

$$
\begin{aligned}
\text { Obs }= & {\left[g, B P, r, G, i, R P, J_{2 \mathrm{M}}, J_{\mathrm{U}}, H_{\mathrm{U}}, H_{12 \mathrm{M}}, K_{2 \mathrm{M}}, K_{\mathrm{U}}, \varpi\right], } \\
\sigma= & {\left[\sigma_{\mathrm{g}}, \sigma_{\mathrm{BP}}, \sigma_{\mathrm{r}}, \sigma_{\mathrm{G}}, \sigma_{\mathrm{i}}, \sigma_{\mathrm{RP}},\right.} \\
& \left.\sigma_{J_{2 \mathrm{M}}}, \sigma_{J_{\mathrm{U}}}, \sigma_{H_{\mathrm{U}}}, \sigma_{H_{2 \mathrm{M}}}, \sigma_{K_{2 \mathrm{M}}}, \sigma_{K_{\mathrm{U}}}, \sigma_{\varpi}\right],
\end{aligned}
$$

where the $2 \mathrm{M}$ subscript stands for 2MASS and U for UKIDSS. The photometric uncertainties are the combination of their standard measurement uncertainty, and a systematic error. The value of the latter is taken as 0.03 mag for $g, r$, and $i$ (Barentsen et al. 2014; Drew et al. 2014), $0.01 \mathrm{mag}$ for $G, G_{\mathrm{BP}}, G_{\mathrm{RP}}$ (Riello et al. 2020), $0.03 \mathrm{mag}$ for $J_{2 \mathrm{M}}, 0.02$ for $H_{2 \mathrm{M}}$ and $K_{2 \mathrm{M}}$ (Skrutskie et al. 2006), and $0.03 \mathrm{mag}$ for $J_{\mathrm{U}}, H_{\mathrm{U}}, K_{\mathrm{U}}$ (Hodgkin et al. 2009).

The best-fitting model will thus be that which minimizes the negative logarithm of the likelihood:

$$
\ln (P(O b s \mid \theta))=-\frac{1}{2} \sum_{i}^{n} \frac{(\operatorname{Obs}(i)-\operatorname{Model}(i))^{2}}{s_{i}^{2}}+\ln \left(s_{i}\right),
$$

To explore the posterior distribution and identify the bestfitting parameters, we use the emcee MCMC package in PYTHON (Foreman-Mackey et al. 2013). For the MCMC simulation, we use 1000 walkers, which undergo 100 burn-in iterations and then 1000 iterations to fully sample the posterior distribution. The fitted parameters are taken as the median of the resulting posterior distribution, while the 16th and 84th percentile values provide the lower and upper $1 \sigma$ bounds. We also extracted fits and uncertainties for the intermediate parameters, $\log \left(T_{\text {eff }}\right)$ and $\log ($ Lum $)$. The median of the posterior distribution was preferred over the best-fitting value for all of these quantities, as it showed better agreement for objects with spectroscopic effective temperatures (see Section 3.2.4). An example of the posterior distributions, SED fit and posterior distributions of effective temperature and luminosity in the HR diagram are shown in Fig. 4.

\subsubsection{General results}

SED fits were performed for 20498 objects and the distribution of the physical parameters is displayed in Fig. 5.

The distribution of $T_{\text {eff }}$ shows that the sample is clearly dominated by cool red giants, despite our previous cuts, but we also find that 4680 objects have $\log \left(T_{\text {eff }}\right)>4$ (OB stars, 22.8 per cent), and 818 have $\log \left(T_{\text {eff }}\right)>4.3$ (O stars, 4.0 per cent). The distributions of $\log \left(L / L_{\odot}\right)$ and $\log \left(M / \mathrm{M}_{\odot}\right)$ reveal, respectively, peaks between 10 and $100 L_{\odot}$ and between 1 and $3 \mathrm{M}_{\odot}$, another strong indicator of the dominance of cool giants.

As expected from our distance cuts, most of our fitted distances fall between 1 and $2.5 \mathrm{kpc}$, with a peak between 1.5 and $2 \mathrm{kpc}$. Sources fitted close to the upper prior on distance $(5 \mathrm{kpc})$ are often indicators of poor fits. The reddening for our fitted objects, as derived from the reddening maps of Green et al. (2019) shows a broad spread from $A_{V}=0$ to 6 . 

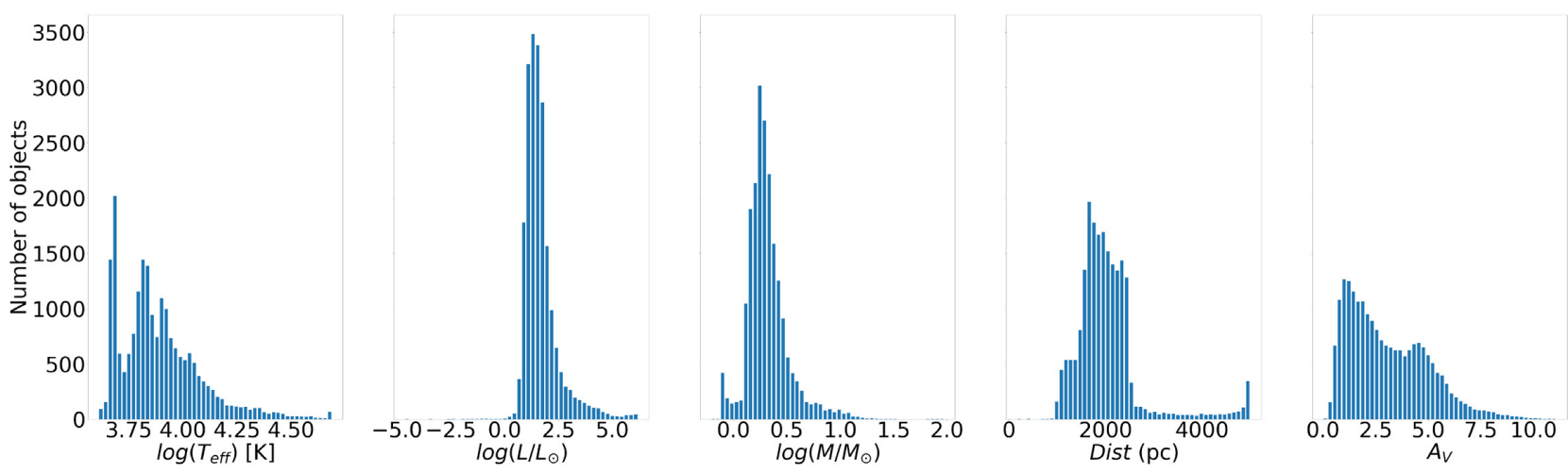

Figure 5. Distribution of the fitted physical parameters derived from the SED-fitting process for the 20498 sources.

\subsubsection{Comparison with known results}

We cross-matched our sample with the Simbad database with a matching radius of 1 arcsec and found 2672 matches. From this list, we focus only on those with a clearly identified spectral type, a reference, and with a quality measurement of 'A', 'B' or 'C' ('D' and ' $E$ ' are considered too low quality).

We determine the effective temperatures from the spectral type using the tabulations from Martins, Schaerer \& Hillier (2005) for the O-type stars (observed scale), from Trundle et al. (2007) for early B-type stars, from Humphreys \& McElroy (1984) for late B-type stars of luminosity classes 'I' or 'III' and from Pecaut \& Mamajek (2013) for the others, interpolating between these tabulations where they overlapped. We required at least a spectral type and a spectral subclass, used the spectral type of the primary star for binaries, assumed a luminosity class of ' $\mathrm{V}$ ' when not specified, interpolated for luminosity classes 'II' and 'IV', and chose error bars of one spectral subclass. This process reduces the number of stars with spectroscopic effective temperatures to 205 objects, including 44 from Blaha \& Humphreys (1989) and 60 from Berlanas et al. (2019).

Fig. 6 shows a comparison between the spectroscopic effective temperatures and those from our SED fits, and reveals that very few cool stars are identified as hot stars by our SED-fitting process. We define our recovery rate as $R R=\frac{T P}{T P+F N}$, where $T P$ stands for the number of true positives and $F N$ corresponds to the number of false negatives. Our recovery rate is 97 per cent $(96,92$, and 86 per cent) for stars with SED-fitted $\log \left(T_{\text {eff }}\right)>4.0(4.1,4.2,4.3)$, and our contamination rate $\left(\frac{F P}{T P+F P}\right.$, where $F P$ is the number of false positives) is between 3 and 14 per cent, depending on the temperature threshold used.

Exploring our fits in more detail, we found that sources with a large extinction $\left(A_{V}>4 \mathrm{mag}\right)$ typically have higher $\ln (f)$ values $(>-5)$ and that these corresponded to stars with poorer fitted temperatures (when compared to literature values) and larger uncertainties on the fitted quantities. As an example of this, if we focus our comparison on the 44 objects from Blaha \& Humphreys (1989) with literature spectral types (typical extinction of $A_{V}=1-2$ mag), we find a recovery rate of 92-100 per cent, while if we limit our comparison to the 60 objects in Cyg OB2 (typical extinction of 4-5 mag) listed by Berlanas et al. (2019) our recovery rate drops to 85-95 per cent, slightly inferior. Fortunately, our focus is not on the high-extinction region of $\mathrm{Cyg} \mathrm{OB} 2$, but on the other OB associations that exhibit a lower extinction, meaning that our recovery rate for OB stars is approximately $92-100$ per cent.

Finally, we explored the impact of using a single value of $\log (g)$ in our fitted stellar spectral models. This was necessary because

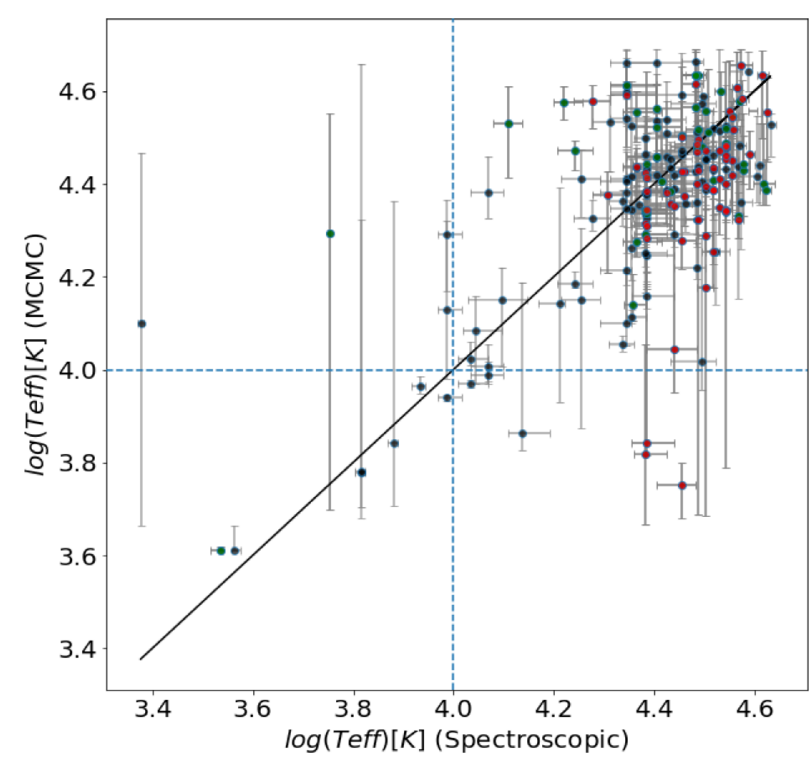

Figure 6. Comparison between the literature spectroscopic temperature with that from our SED fits, for the 205 objects with reliable spectral types. Blue dashed lines correspond to the 'limit' at $\log \left(T_{\text {eff }}\right)=4$ (dividing OB stars from cooler stars). Green sources are from Blaha \& Humphreys (1989), while red ones are from Berlanas et al. (2019).

of the difficulty finding models covering the full range of $T_{\text {eff }}$ and $\log (g)$, and with a sufficient wavelength coverage. We estimated the bias that using a fixed value of $\log (g)$ introduced by comparing the effective temperatures and luminosities derived from our SED fits with those derived spectroscopically, for the 199 sources with spectroscopic surface gravities. We divided this sample into stars with low $(\log (g)<3.5)$ and high $(\log (g)>3.5)$ surface gravity. We found that stars with a low surface gravity had their SED-fitted effective temperatures overestimated by $\sim 0.1 \mathrm{dex}$, while their luminosities were underestimated by $\sim 0.01 \mathrm{dex}$. Both of these effects will cause evolved stars with low surface gravities to be fitted closed to the ZAMS than they should be, underestimating their ages.

\section{IDENTIFYING NEW OB ASSOCIATIONS}

In this section, we exploit our sample of $\mathrm{OB}$ stars to search for $\mathrm{OB}$ associations in the Cygnus region. For this purpose, we will use a new method, the results of which will be compared with existing clustering algorithms. 

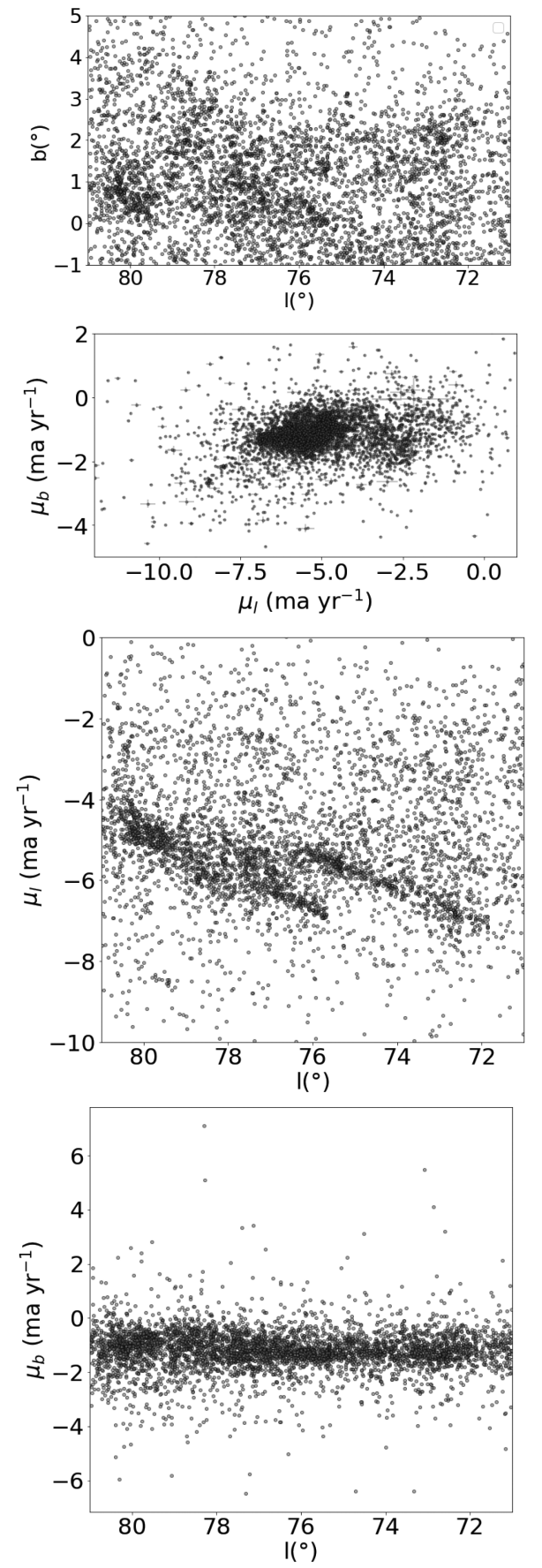

Figure 7. Spatial and proper motion distributions of the 4680 objects fitted with $\log \left(T_{\text {eff }}\right)>4$.

\subsection{Broad kinematics distribution of OB stars}

Fig. 7 shows the broad spatial and kinematic distribution of our SEDfitted OB stars $\left(\log \left(T_{\text {eff }}\right)>4\right)$. The spatial distribution (top panel) shows a broad density gradient as a function of Galactic latitude, as expected, with multiple overdensities that might represent $\mathrm{OB}$ associations (including one in the position of Cyg OB2 at $l \sim 80^{\circ}, b$ $\sim 1^{\circ}$ ). The proper motion distribution (second panel) shows that the bulk of the stars have proper motions between $\mu_{1}=-8$ and -2 mas $\mathrm{yr}^{-1}$ and around $\mu_{\mathrm{b}} \sim-1 \mathrm{mas}^{-1}$. The graph displaying $l$ versus $\mu_{l}$ (centre panel) reveals two diagonal structures, above and below $l=76^{\circ}$, an interesting saw-tooth pattern indicating a correlation between position and velocity that is usually indicative of some form of expansion (we will return to this feature on Section 5). No such feature was observed in $\mu_{\mathrm{b}}$ (see the bottom panel of Fig. 7).

The expected motion of stars in this sightline can be estimated using a simple Galactic rotation model. Eilers et al. (2019) determined a Galactic rotation curve of $v=229 \mathrm{~km} \mathrm{~s}^{-1}-1.7 \mathrm{~km} \mathrm{~s}^{-1} \mathrm{kpc}^{-1}(R-$ $\left.R_{\odot}\right)$. Along our line of sight these stars have a Galactocentric radius of $R \sim 7.7 \mathrm{kpc}$, if we assume $d=1.8 \mathrm{kpc}$ and $l=72^{\circ}$, at an angle intercepting the line of sight of 4.5 , we have a rotation speed of $229.6 \mathrm{~km} \mathrm{~s}^{-1}$. This equates to a motion of about $-18 \mathrm{~km} \mathrm{~s}^{-1}$ in the $l$ direction. With the local Solar motion of $\mathrm{U}=11.1 \mathrm{~km} \mathrm{~s}^{-1}$ and $\mathrm{V}=12.24 \mathrm{~km} \mathrm{~s}^{-1}$ (Schönrich, Binney \& Dehnen 2010), the Sun requires a correction of about $-6.73 \mathrm{~km} \mathrm{~s}^{-1}$ in that direction. This therefore results in an apparent motion of $-24.73 \mathrm{~km} \mathrm{~s}^{-1}$, or a

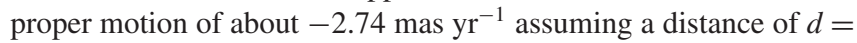
$1.8 \mathrm{kpc}$. The majority of stars in this direction have a proper motion in $l$ more negative than this, suggesting that the Cygnus region is moving towards lower $l$ values (towards the inner Galaxy) relative to other stars orbiting within the Milky Way.

Stars in this sightline appear to have a typical proper motion in Galactic latitude of -1 mas $\mathrm{yr}^{-1}$. If the motion of the Sun is $7 \pm 0.5 \mathrm{~km} \mathrm{~s}^{-1}$ (Bland-Hawthorn \& Gerhard 2016). In the positive $b$ direction, then stars in the Galactic disc will have negative proper motions of a similar magnitude. At a distance of $1.8 \mathrm{kpc}$, this would

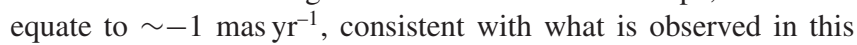
sightline.

\subsection{Identifying kinematic groups}

For the purpose of identifying $\mathrm{OB}$ associations, we limit our sample of stars to those with $\log \left(T_{\text {eff }}\right)>4.2$ (approximately equivalent to spectral type B5 or earlier), which gives us 1349 stars. This limits us to a sample of more massive and therefore younger stars with which to identify young groups.

To identify $\mathrm{OB}$ associations or groups of kinematically coherent stars within the wider distribution of OB stars, we seek to find groups of stars relatively close together on the sky whose kinematics are more similar to each other than to the wider distribution of OB stars. To start, we want to visualize how the relative kinematics of stars changes over our area of study. To do this, we define a grid with a cell size of 0.1 and at each point in this grid, we select the ten nearest stars from the sample of 1349 stars. We then perform a KolmogorovSmirnov (KS) test comparing the proper motion distribution of these ten stars with the proper motion distribution of all 1349 stars. We do this for both the proper motion in $l$ and in $b$, obtaining a $p$-value for each, and then multiply them together. The result is the probability that the kinematics of stars in that area are consistent with that of the wider population.

Fig. 8 shows the distribution of this probability, over our area of study, effectively highlighting regions that are kinematically distinct compared to the wider area. Contours are shown at $2 \sigma, 3 \sigma$, and $4 \sigma$ significance. Immediately apparent is that there are a number of areas with very significantly distinct kinematics $(\log (P)<-6)$, one of which is located in the direction of Cyg OB2 $\left(l=80^{\circ}, b=+1^{\circ}\right)$, giving an early verification of our method.

To test our method and the significance of our results, we perform a simple experiment, repeating our analysis 100 times, but in each iteration randomizing the proper motions of all the stars and calculating the $\log (\mathrm{P})$ value at each position. The goal of this process is to check whether the probabilities derived from the actual proper motions are consistent with a random distribution of kinematics. Fig. 9 compares the observed distribution of probabilities with that 


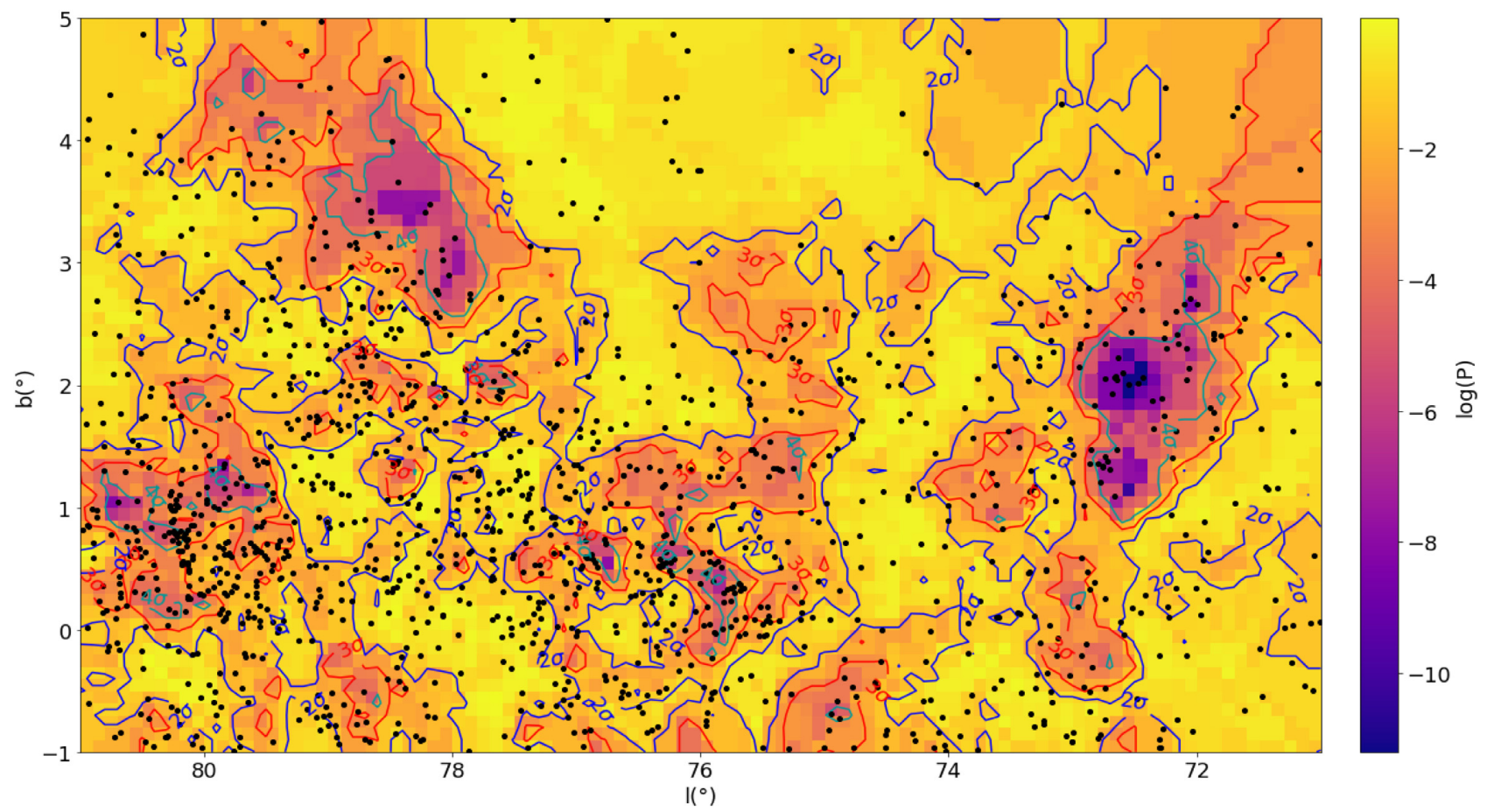

Figure 8. The Cygnus region, colour-coded according to probability, $\log (\mathrm{P})$, that the kinematics of stars in that vicinity are consistent with that of the wider population. The $2 \sigma, 3 \sigma, 4 \sigma$ contours represent levels at $\log (\mathrm{P})=-1.3,-2.6$, and -4.2 , respectively.

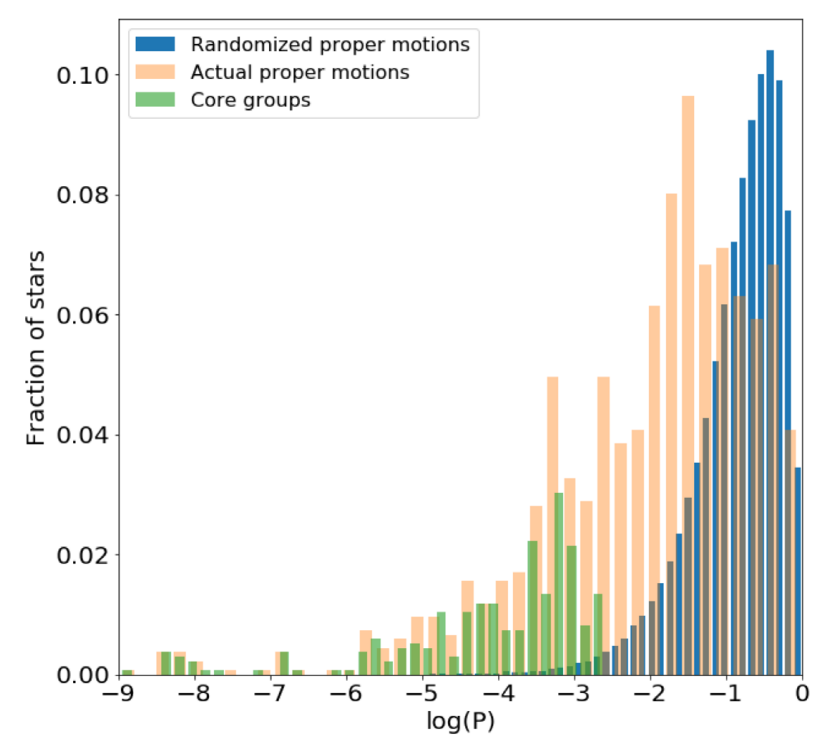

Figure 9. $\log (\mathrm{P})$ values across our survey area deduced from the randomized proper motions, from the actual proper motions, and for the core groups (before removal of outliers).

derived from the randomized velocities and shows the former reaches considerably smaller values of $\log (\mathrm{P})$. This suggests that the majority of the kinematic structures observed in Fig. 8 are likely to be real.

To identify stars in a given group, we choose the $3 \sigma$ contour level and define all stars falling within such a contour as being tentatively part of that group. Given the number of contours, we required each group to contain a minimum of 10 stars, which led to the identification of five groups of stars with similar kinematics. This method of identifying groups is likely to include some contaminants that are projected against real groups. To exclude any such contaminants we then calculated the median and 16th and 84th percentile values of $\mu_{l}, \mu_{b}$ and parallax, and excluded sources that were more than $3 \sigma$ from the median value in each dimension, where $\sigma$ is the true dispersion calculated using the outlier-resistant method from Ivezić et al. (2014). This process reduced the membership of each group by approximately 20-30 per cent.

From this, we find the following groups:

(i) Group A is located in the upper right-hand part of Fig. 8, with $l=71.5-73^{\circ}$ and $b=0.5-3.0$.

(ii) Group B spans the upper left-hand part of Fig. 8, in the region $l>77-80^{\circ}$ and $b=2.5-5.0$.

(iii) We initially defined a third group in the region of 1 from 75 to 77.5 and $b$ from -0.5 to $2^{\circ}$, but further investigation (see later) suggested this constituted two groups with distinct $\mu_{l}$ distributions and so this was separated into two groups, $\mathrm{C}$ and $\mathrm{D}$, with $\mu_{l}$ less than

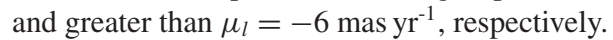

(iv) The group in the lower left-hand part of Fig. 8 corresponds to Cyg OB2 and its surrounding. It forms group E.

Our initial selection of group members is based on stars with $\log \left(T_{\text {eff }}\right)>4.2$, chosen as the balance between a large enough sample and stars that are sufficiently hot to be very massive and young. With our groups defined, we can now expand their membership by considering the inclusion of slightly cooler stars. We add stars that fulfill either $\log \left(T_{\text {eff }}\right)>4$ (and $<4.2$ ) or $\log \left(\frac{L}{L_{\odot}}\right)>2.5$, hence we catch all possible $\mathrm{OB}$ stars, and massive post-main-sequence stars that have evolved to cooler temperatures. We add to each group any star that fulfils these criteria, falls within the same $3 \sigma$ contours, and is within two standard deviations of the median value of parallax and proper motion (with the median and standard deviations defined from the current group membership). This increased the membership of the existing groups to a total of $123,93,93,86$, and 163 stars. 


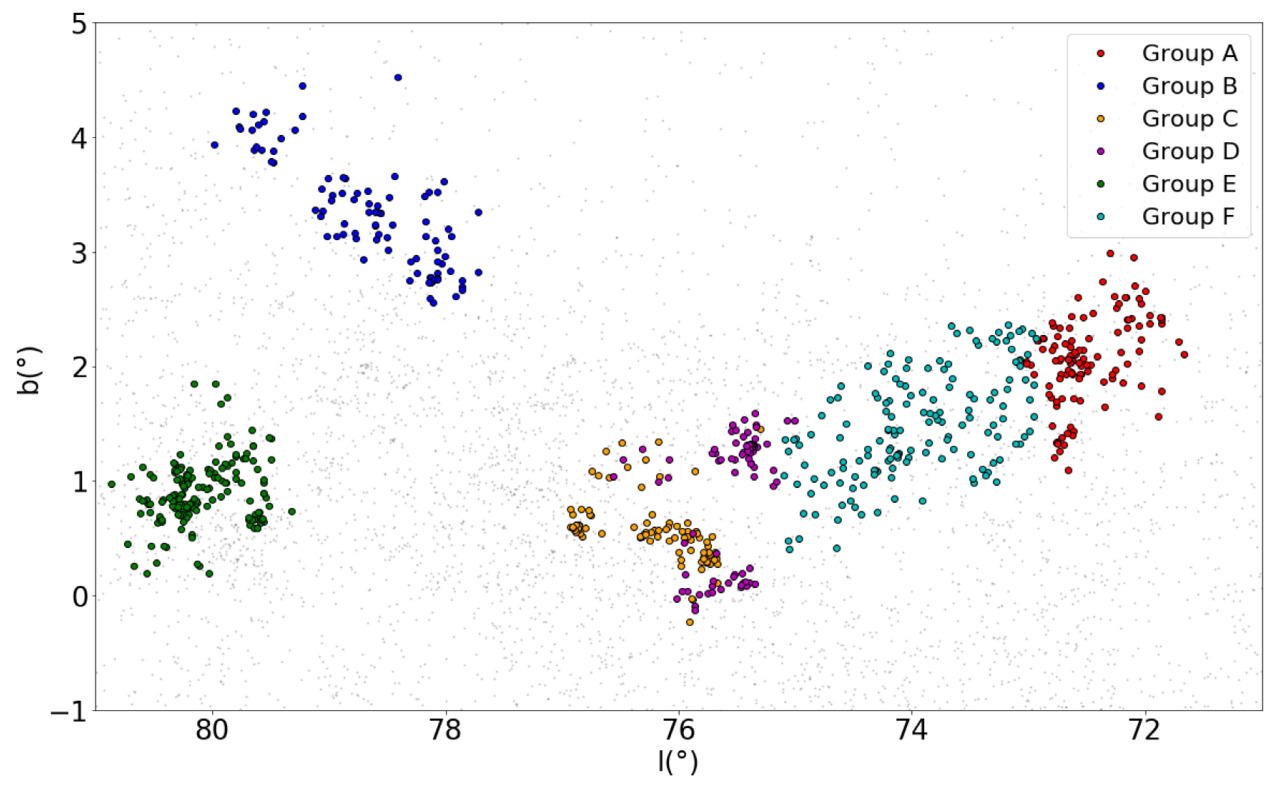

Figure 10. Spatial distribution of all the identified new OB groups (coloured symbols), plotted against the field OB stars (black dots).

Finally, in later analysis of the kinematics of these stars, we became aware of a 'gap' between groups $\mathrm{A}$ and $\mathrm{D}$ in their distributions in $l$, $b$, and $\mu_{l}$. We identified the stars that fell between groups A and D in both $l$, but also in the $l$ versus $\mu_{l}$ diagram (see Fig. 11), trimmed the sample and then added in slightly cooler stars, all as described above (this time also discarding the outliers in $l$ and $b$ ). This resulted in a group of 155 stars. It should be noted that the group has been selected manually, contrary to the other groups. Fig. 10 shows the spatial distribution of all the final groups, while Fig. 11 shows the proper motion distribution (left-hand panel) and $l$ versus $\mu_{l}$ (righthand panel) distribution of the groups.

\subsection{Comparison with historical associations}

How do our new groups compare to the historical associations discussed in Section 2? We compared the membership of our groups with that of the historical associations, our results confirming what we found in Section 2: while Cyg OB2 stands out as a real group (corresponding roughly to our group E) and Cyg OB3 has some overlap with group A, the same is not true for the other groups, which show very little overlap with the historical OB associations (typically 5-20 per cent of the historical association members are found in the nearest new groups we identify). Comparison plots between the historical associations and our new groups are shown in Fig. A1.

\subsection{Comparison with the results from DBSCAN}

To test our group-finding method and compare the results to those from modern clustering algorithms, we again selected the 1349 stars of our sample with $\log \left(T_{\text {eff }}\right)>4.2$ and used DBSCAN (Pedregosa et al.2011) to identify groupings, choosing the number of nearby stars to be ten for similarity with our own method and using a maximum normalized distance between stars (in both spatial and proper motion coordinates) to be considered part of the same group of 0.05 .

DBSCAN was able to identify six groups, respectively containing 158, 167, 23, 52, 12, and 32 stars. Fig. A2 shows how these groups compare to our groups, some of which match up well, while others do not. For example, our group A agrees well with DBSCAN group 6 and our group E matches with DBSCAN group 2, though in both cases our groups are more extended than those identified by DBSCAN. In other cases, the agreement is less good, our groups $\mathrm{C}$ and D overlap with DBSCAN groups 1 and 3 in a complex manner. The most obvious distinction lies between group 5 from DBSCAN and our group B. DBSCAN was unable to pick out the bulk of group B. This is because, as outlined above, one of the main parameters of DBSCAN is the relative distance between the objects used to form clusters. Consequently, since the same value was used throughout the entire region, it was impossible to detect this lower density group, while still detecting the higher density groups found elsewhere. Our method, on the other hand, only relies on the number of neighbours stars, and therefore is not biased by density in this regard.

Sometimes the opposite trend is observed, i.e. some stars have been identified as parts of the groups in DBSCAN but not with our method. This stems from our selection: we chose sources within $3 \sigma$ contours, rejecting sources that fell outside of these contours and which may represent members in the periphery of a group.

\section{ANALYSIS OF THE NEW OB ASSOCIATIONS}

In this section, we analyse the physical and kinematic properties of our new groups. To do so, we calculate several of their properties and produce HR diagrams of their members in order to obtain broad estimates of their age. We then give a few words about their dynamics. Table 1 summarizes some of the physical and kinematic properties of each group, which were calculated as follows.

\subsection{Physical properties of the individual groups}

To estimate the observed number of $\mathrm{O}$ and $\mathrm{B}$ stars in each group, we defined B-type stars as those with $\log \left(T_{\text {eff }}\right)>4$ and $\log \left(T_{\text {eff }}\right)<4.47$ and O-type stars as those with $\log \left(T_{\text {eff }}\right)>4.47$. Where a spectral type is available from the literature we use the spectroscopic $T_{\text {eff }}$, otherwise we use the effective temperature derived from our SED fits. The uncertainties on the number of $\mathrm{O}$ and $\mathrm{B}$ stars was estimated 

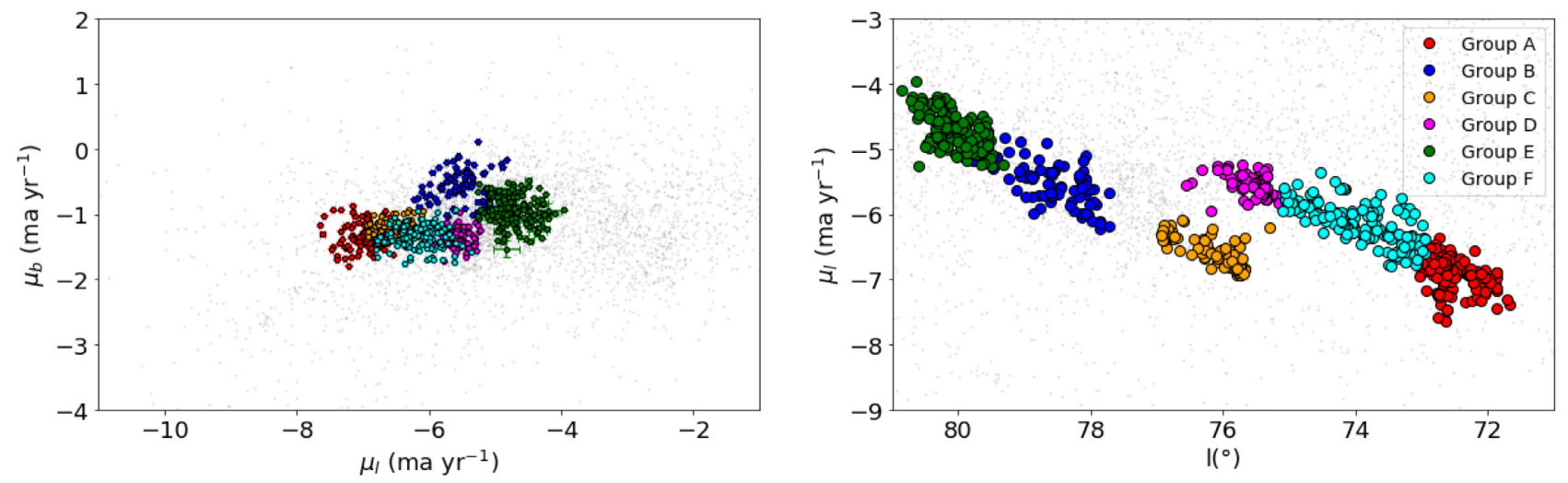

Figure 11. Left-hand panel: proper motion distribution of all six new OB groups (coloured symbols), plotted against the field OB stars (black dots). Right-hand panel: proper motion in $l$ plotted against $l$ for the same objects. The uncertainties in $\mu_{l}$ are comparable to, or smaller than, the symbol size, and thus are not shown.

Table 1. Properties of our new OB associations. The first column indicates the parameter, where the subscript ' $m$ ' indicates the median value and ' $\sigma$ ' the dispersion.

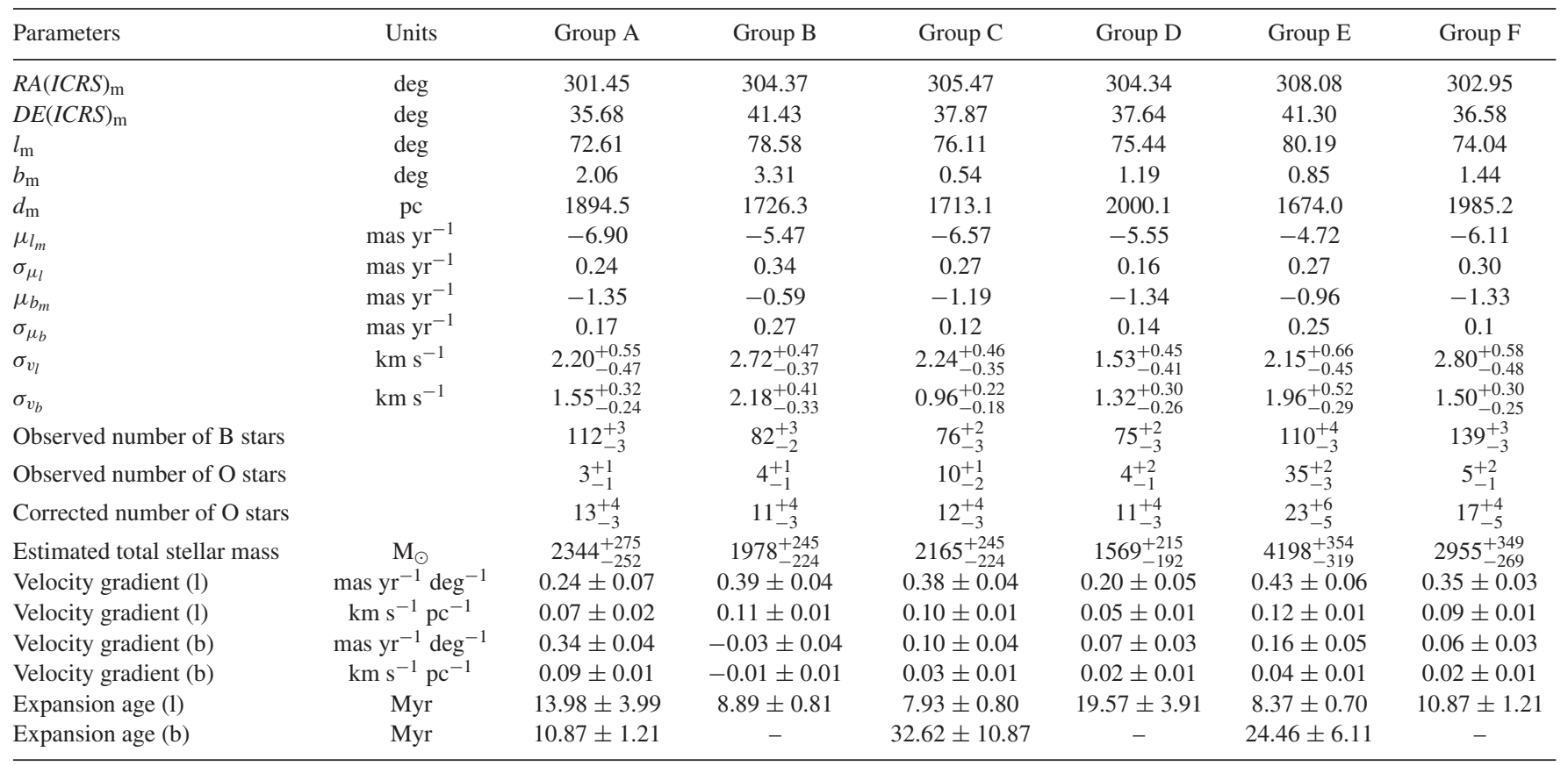

using a Monte Carlo experiment, varying the temperature of each star according to its uncertainties.

We then attempt to calculate the total mass of each group. We used the SED-fitted masses for consistency. To determine the complete mass range, we inspected the mass function, chose the turnover point of the mass function as the lower limit and the post-main-sequence turn-off mass at $15 \mathrm{Myr}$ for the upper limit (Ekström et al. 2012). Based on this, we estimate our samples to be relatively complete in the mass range of $3.2-13.8 \mathrm{M}_{\odot}$.

The filtering of the data we used (Section 3.1) means that our samples of members will be slightly incomplete. We estimate our incompleteness by calculating the fraction of stars, as a function of magnitude, that were discarded at each step in Section 3.1. The completeness level varies for each step, and as a function of magnitude (shown in Fig. 12), with cumulative completeness of 7080 per cent in our magnitude range of interest. Fig. 12 shows these incompleteness curves and an example of how the membership of one of our groups increases once this incompleteness is accounted for.
The last source of incompleteness stems from the false positive and negative rates from our SED-fitting process. In Section 3.2.4, we quantified the recovery rates at different temperature thresholds by comparing our SED-fitted temperatures with literature spectroscopic temperatures (see Fig. 6). We repeat this here using as a threshold the minimum mass of our complete mass range $\left(\log \left(T_{\text {eff }}=4.11\right)\right.$. For sources with $A_{V}<4$ (appropriate for the low-extinction associations), our false negative rate is 5.0 per cent and our false positive (contamination) rate is 5.9 per cent, while for sources with $A_{V}>4$ (appropriate for Cyg OB2), these quantities are equal to 3.5 per cent and 0.0 per cent, respectively. We then estimate the 'true' number of stars, $T$, from:

$T=\frac{N}{1-F}-N P$

where $N$ is the corrected number of stars within the complete mass range, $F$ is the false negative rate and $P$ is the false positive rate. 


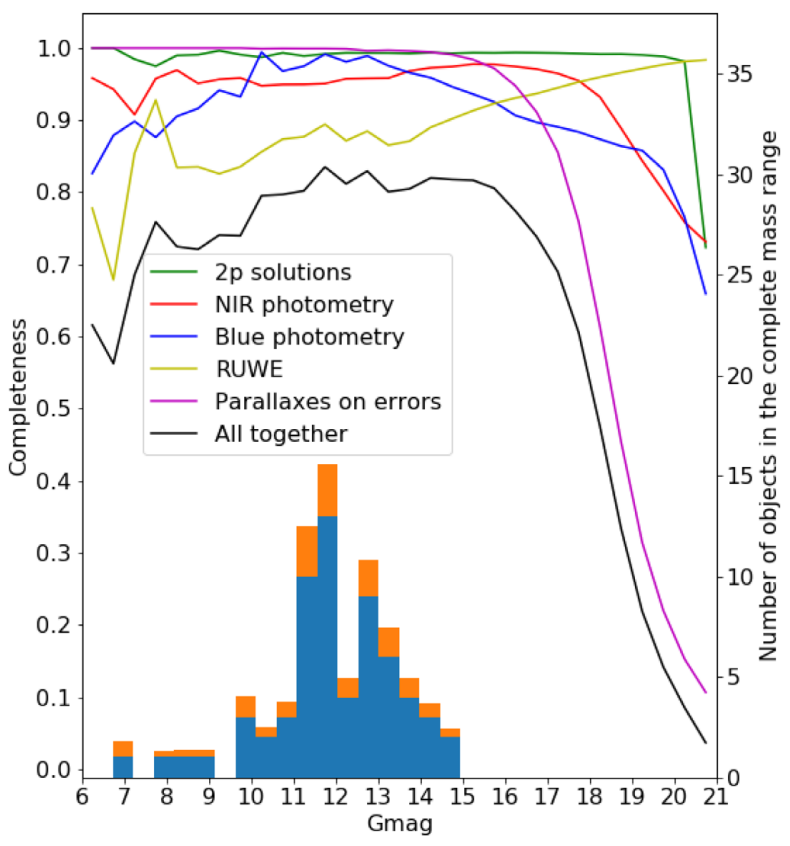

Figure 12. Completeness as a function of $G$ magnitude for the various data filtering steps in Section 3.1. The black curve corresponds to the convolution of all effects combined together. The histogram show the number of stars in group A within the complete mass range both before (blue) and after (orange) applying the incompleteness corrections.

To estimate the total mass of each group, we apply both of these corrections. Subsequently, to estimate the total mass of each group we perform a Monte Carlo simulation using the mass functions from Maschberger (2013) to sample stellar masses, counting the number of stars in our representative mass range as well as the total number (and mass) of stars. When our simulation reaches the total number of observed stars in our representative mass range we halt the simulation and take the total mass of the group of stars. We repeat this 10000 times, varying the number of stars observed in the representative mass range (according to their uncertainties), to estimate the uncertainties on the total mass. We use this same method to deduce the incompleteness corrected number of O-type stars, also listed in Table 1.

\subsection{HR Diagrams and ages of group members}

HR diagrams for all the groups are shown in Fig. 13 using spectroscopic (where available) or SED-fitted (otherwise) effective temperatures and luminosities derived using the fitted distances, extinctions, $K$-band magnitudes and bolometric corrections (the latter from Jordi et al. 2010).

Estimating the ages of these groups is difficult. Many of the groups include members close to the ZAMS at high luminosities, implying the existence of particularly massive (and therefore young) stars. These would possibly need to be as young as 3-5 Myr, depending on initial rotation rate. There are notable examples of this in groups $\mathrm{B}, \mathrm{C}$ and $\mathrm{E}$ (Cyg OB2). Some of the groups also include members moving away from the main sequence, particularly around the 10Myr isochrone, implying the presence of stars around this age. Examples of this can be found in groups A, D, E (to a lesser extent), and $\mathrm{F}$.

Using these indicators of age we can place the groups in an approximate age order, with groups $\mathrm{B}, \mathrm{C}$ and $\mathrm{E}$ as the youngest (due to the presence of very luminous stars within them), and groups $\mathrm{A}, \mathrm{D}$, and $\mathrm{F}$ as the oldest (due to the presence of stars at or beyond the $10-\mathrm{Myr}$ isochrone). However, we note that many OB associations, including these, exhibit large age spreads (see e.g. Wright 2020) and therefore assigning a single age to any association is difficult.

\subsection{Kinematics of the individual groups}

We calculated the median positions and proper motions of each group, along with their distance. The proper motion dispersions were computed using the method from Ivezić et al. (2014), again with random Gaussian sampling to estimate uncertainties. Velocity dispersions range between 1 and $3 \mathrm{~km} \mathrm{~s}^{-1}$, as indicated in Table 1, consistent with other OB associations (Wright 2020).

To determine whether the groups are expanding, we search for evidence of velocity gradients in the kinematics of our stars. To compute the velocity gradients, we fit a linear relationship using an MCMC simulation and the emcee package, and obtain velocity gradients for each group (see Table 1). An example of a fit is shown in Fig. 14. We note that the velocity gradients are generally anisotropic and larger in the $l$ direction compared to the $b$ direction, similar to the pattern of anisotropic expansion observed in other OB associations such as Sco-Cen (Wright \& Mamajek 2018).

Velocity gradients can then be converted into expansion ages, listed in Table 1. Expansion ages are based on the assumption that the entire group expanded from a compact region of space, and therefore represent upper limits rather than precise ages. We have not calculated these quantities in Galactic latitude for the groups B, $\mathrm{D}$ and $\mathrm{F}$ as their velocity gradients are either too small or negative (both of which result in unphysical expansion ages). On the other hand, results for the $l$ direction tend to be closer to the likely ages for these systems. The expansion ages in the $l$ and $b$ directions do not agree for any of the groups except group A, as has been observed in many other OB associations (see e.g. Wright 2020).

While these expansion ages should be considered as upper limits, it is reassuring to see that the three groups we identified as being the youngest (B, C, and E, see Section 2) have the smallest expansion ages, and the three older groups have the largest expansion ages.

\subsection{Large-scale dynamics of the Cygnus associations}

In the right-hand panel of Fig. 11, the proper motion in Galactic longitude is plotted against Galactic longitude. A very clear trend of increasing proper motion is shown as a function of Galactic longitude for the stars in two combinations of associations: groups A, D, and F at low longitudes and groups B, C, and E at high longitudes. Notably, these two sets correspond to the three young groups and the three old groups (Section 5.2), hinting at a connection in both dynamics and age. This pattern repeats itself on a scale of five degrees in longitude (from $l=81^{\circ}-76^{\circ}$ and from $l=76^{\circ}-71^{\circ}$ ). and approximately 3 mas $\mathrm{yr}^{-1}$ in proper motion. At a rough distance of $1800 \mathrm{pc}$, these equate to $\sim 150 \mathrm{pc}$ and $\sim 25 \mathrm{~km} \mathrm{~s}^{-1}$, respectively. No such pattern is seen for $\mu_{b}$ as a function of either $l$ or $b$.

\section{DISCUSSION}

In this section, we discuss our results, how they compare to previous studies and what they imply for our general understanding of $\mathrm{OB}$ associations. Our main results are as follows:

(i) Most of the historical OB associations in the Cygnus region lack kinematic coherence and therefore do not appear to be genuine 

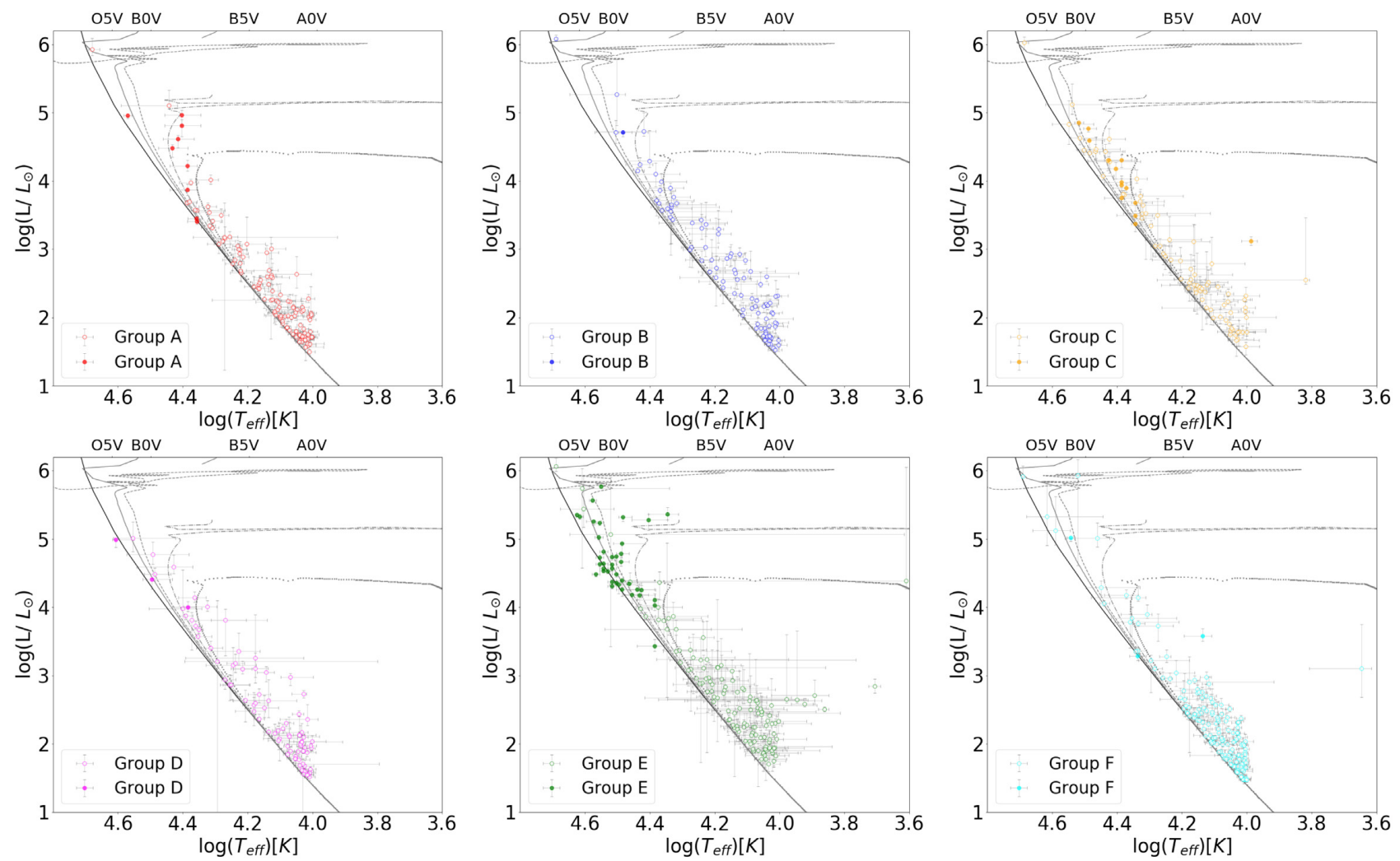

Figure 13. HR diagrams for all the groups. Full coloured symbols correspond to those with spectroscopy while the empty symbols are those without. Isochrones has been drawn from the rotational models in Ekström et al. (2012). From the top to bottom panels, these are the 3.16-, 5-, 10-, and 20-Myr isochrones. Positions of some spectral types have been indicated on the top horizontal axis for clarity.

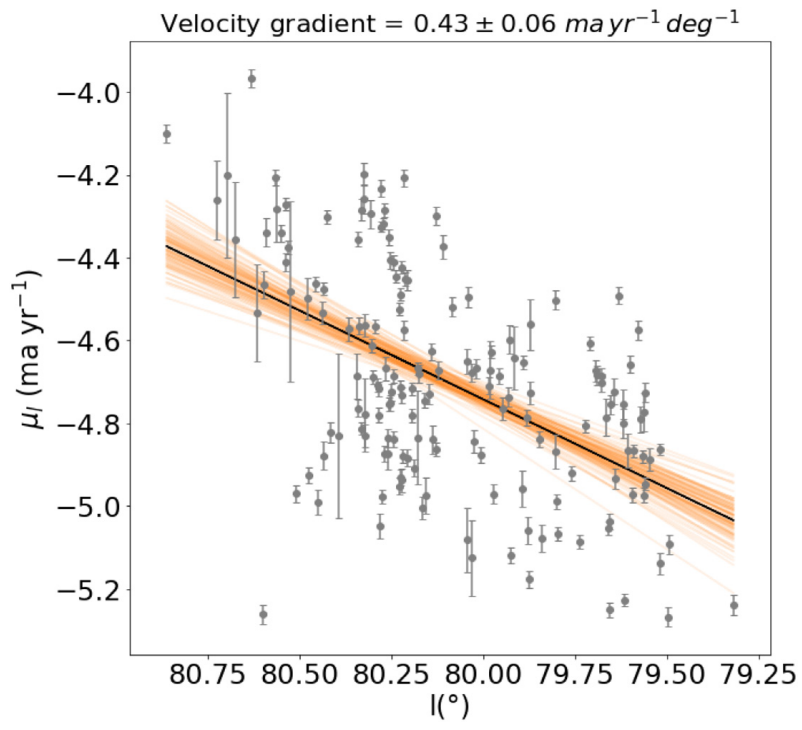

Figure 14. Results of the MCMC fit between position and proper motion for the $l$ direction for group E. In total, 100 random samples have been drawn from the posterior distribution. The black line corresponds to the best-fitting value for the velocity gradient which has been indicated on the top of the plot.

OB associations. We identified six new systems that we compared to the previous divisions. There is a strong overlap between our group $\mathrm{E}$ and Cyg OB2, a partial overlap between group A and Cyg OB3, but no significant overlap between our other groups and the other historical associations. (ii) We calculated the broad properties of each new association, including estimates of their total stellar mass, quantifying their incompleteness. Group E (Cyg OB2) contains by far the largest number of O-type stars and is the most massive.

(iii) We calculated the velocity dispersions of each group and searched for evidence that they are expanding. We found strong evidence of expansion for all of the groups in the $l$ direction and in groups $\mathrm{A}, \mathrm{C}$, and $\mathrm{E}$ in the $b$ direction.

(iv) We discovered a correlation between $l$ and $\mu_{l}$ on large scales across the entire Cygnus region, specifically connecting the three oldest and the three youngest groups, thus showing a connection between age and dynamics.

\subsection{The new Cygnus OB associations}

We have identified six association-like groups in Cygnus, each of which has a total mass of between 1500 and $4500 \mathrm{M}_{\odot}$. These groups are remarkably kinematically-coherent (see Figs 10 and 11), especially when compared to the historical OB associations (Section 2). Comparing these groups to the historical associations, the best match is between Group E and Cyg OB2 with 64 stars in common, yet our group has a better kinematic coherency.

Zari et al. (2021) provided a map of hot and luminous OBA stars in the local part of the Milky Way and noticed that massive starforming regions and $\mathrm{OB}$ associations appeared as overdensities. Yet only Cyg OB2 is clearly prominent in their map, meaning that in spite of their completeness, they were not able to identify some of the groups that we have. Cyg OB2 is the densest and most massive group of the region, while the other groups have a lower density, 
by approximately an order of magnitude, explaining why Zari et al. (2021) did not detect such groups.

Berlanas et al. (2019) divided Cyg OB2 in two substructures along the line of sight, with a main group situated around $1760 \mathrm{pc}$ and a smaller, foreground group at $1350 \mathrm{pc}$, with group E matching the former one. Orellana, De Biasi \& Paíz (2021) refined the census of the region by identifying 2767 proper motion members of Cyg OB2 (with a mean distance of $1669 \pm 5 \mathrm{pc}$, very close to the median distance of group E in Table 1). The existence of two separates structures composing Cyg OB2 could explain why our group E does not include some of the old classical members of Cyg OB2 and why our estimate of the total mass of group $\mathrm{E}$ is smaller than the mass of Cyg OB2 estimated by Wright et al. (2015). None the less, given their close characteristics, such as central coordinates, distance, total stellar mass, age and high number of O-type stars (Wright et al. 2015, 2016; Berlanas et al. 2019; Orellana et al. 2021), the newly identified stars in group E are likely to be part of Cyg OB2.

\subsection{Expansion}

The expansion of $\mathrm{OB}$ associations has been assumed since their very first discovery as they were argued to be the expanded remnants of dense star clusters (Ambartsumian 1947). Wright (2020) emphasized the two 'extreme' scenarios for the origin of OB associations being hierarchical and clustered star formation. In the latter case, OB associations are formed when star clusters are disrupted by processes such as residual gas expulsion (see e.g. Lada \& Lada 2003), while in the former case multiple structures may form hierarchically and drift apart without a clearly coherent expansion pattern. Which of these two theories can best explain the highly anisotropic expansion patterns now being seen in many $\mathrm{OB}$ associations remains to be seen (though see Kruijssen 2012 for a possible cause of asymmetric expansion).

We measured a clear expansion trend in all of the identified groups in the $l$ direction along with a similar trend in the $b$ direction for the groups A, C, and E. By comparison, Wright et al. (2016) found no clear evidence of expansion in Cyg OB2. Wright \& Mamajek (2018) also failed to detect expansions in both directions in ScoCen, neither did Ward \& Kruijssen (2018) for their 18 studied OB associations. Using Gaia DR2 data, Melnik \& Dambis (2020) only identified expansion in 6 out of their 28 selected OB associations. On the other hand, Cantat-Gaudin et al. (2019) and Armstrong et al. (2020) measured expansion in sub-groups inside the Vela-Puppis region, albeit anisotropic expansion, while Kounkel et al. (2018) made a similar discovery in the substructures of the Orion complex. Our results fall in the second category and share the feature with those studies that the $\mathrm{OB}$ association groups have been defined kinematically. The fact that our group $\mathrm{E}$ is expanding while Wright et al. (2016) did not detect any expansion in the historical Cyg OB2 illustrates this trend: Wright et al. (2016) included some stars in Cyg OB2 with different kinematics and potentially at a different distance (Berlanas et al. 2019).

It is clear that studies that used the historical membership of an OB association have typically failed to find evidence of expansion, while those studies that have redefined the membership using kinematic information have more consistently identified expansion trends.

\subsection{Large-scale kinematics and expansion}

The large-scale kinematics in the right-hand panel of Fig. 11 reveal two patterns with a length of about five degrees in Galactic longitude

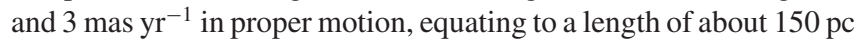

and a velocity of $25 \mathrm{~km} \mathrm{~s}^{-1}$ (at a distance of $1.8 \mathrm{kpc}$ ). The direction of Galactic longitude towards the Cygnus region is approximately transverse to the direction of the Cygnus spur (or spiral arm) within the plane of the Galactic disc. A similar kinematic pattern has recently been observed in the Carina arm by Drew et al. (2021).

What could produce such a large-scale, modulating kinematic pattern amongst these young stars? We explored whether such a kinematic pattern could emerge due to the projection of Galactic rotation, using the rotation law of Eilers et al. (2019), individual distances, and calculating Galactocentric radii for each star. The kinematic signature that results does show a dependence between $l$ and $\mu_{l}$, but it is smaller in $\mu_{l}$ by almost an order of magnitude than that observed, and does not exhibit the sawtooth pattern seen in Fig. 11. This implies that the observed kinematic pattern originates not from Galactic rotation but from the local dynamics in Cygnus.

A correlation between distance and velocity in the same direction is often an indication of expansion, which can arise due to feedback. Chevance et al. (2020) have argued that feedback within H II regions promotes velocities of order $\sim 15 \mathrm{~km} \mathrm{~s}^{-1}$, which would likely occur in opposite directions and thus lead to a kinematic pattern with a magnitude of $\sim 30 \mathrm{~km} \mathrm{~s}^{-1}$, very similar to the $25 \mathrm{~km} \mathrm{~s}^{-1}$ pattern we observe. Chevance et al. (2020) observe this phenomena in other galaxies on scales of 100-300 pc, again consistent with the scale of $160 \mathrm{pc}$ we observe. This could indicate that the kinematic pattern we are observing was introduced by a previous generation of stars that exerted feedback on the surrounding gas clouds, that then went on to form the stars we have studied here, with those stars inheriting the motion of the gas. We note also that our six new OB associations are arranged in this large-scale kinematic pattern in groups of three OB associations. From analysis of the HR diagrams of these groups, we estimated that the three youngest associations are part of one of these large-scale patterns and the three oldest associations are part of the second pattern. This temporal link lends weight to the idea that a common origin was responsible for these two large-scale structures.

An argument against such an interpretation is that one would naively expect such an expansion signature to be present in the $b$ direction as well as in $l$, yet we do not observe this. This could be because of the restoring force of the Galactic disc inhibiting such expansion, or because the gas clouds expanding in the $b$ direction did not form a new generation of stars, possibly because of the lower density medium they expanded into that did not trigger further star formation.

Alternatively, the kinematic pattern we observe could be due to Galactic shear forces acting on the primordial giant molecular cloud, with the forming stars inheriting this motion. This interpretation has been argued to explain why the expansion of the Sco-Cen association is primarily in the Galactic $Y$ direction (Wright \& Mamajek 2018). However, the time-scales for Galactic shear to act are $\sim 70 \mathrm{Myr}$ (Dobbs \& Pringle 2013), too short to explain the observed kinematics. Further observations and simulations appear necessary to explain this interesting kinematic pattern.

\section{CONCLUSIONS}

In this paper, we have investigated the existence of the $\mathrm{OB}$ associations in Cygnus, concluded that the current associations, on the whole, do not represent kinematically coherent groups of stars, and identified six new groups of OB stars that appear more coherent and are therefore likely to be real OB associations. 
We have identified OB stars using an SED-fitting process, that exploits photometry and astrometry from large-scale surveys and evolutionary models. This allowed us to identify 4680 candidate OB stars, including 818 probable O-type stars, with an estimated reliability of $>90$ per cent. From this sample, and using a new and flexible tool to identify coherent kinematic groups, we identified six new $\mathrm{OB}$ associations in Cygnus. We compared them with the historical $\mathrm{OB}$ associations and found some overlap between Cyg OB2 and Cyg OB3 and our new associations, but very little overlap with any of the other historical associations.

We measured several physical and kinematic properties of these groups and found a good consistency with other OB associations, notably for velocity dispersion, total mass and age. Expansion was identified and measured in the $l$ direction for all the groups and in the $b$ direction for three of them. This result is comparable to other recent studies that have detected expansion in OB associations when they have been identified or characterized using kinematic criteria and contrasting with studies that have used the historical membership of $\mathrm{OB}$ associations and typically failed to find signatures of expansion.

This highlights the importance of revisiting the historical OB associations. It leads not only to new divisions with a higher kinematic consistency, but also to better characterization of the systems and their expansion. This method can easily be applied to other regions or $\mathrm{OB}$ associations to validate the reality of existing systems or identify new ones.

\section{ACKNOWLEDGEMENTS}

The authors would like to thank the anonymous referee for many helpful suggestions that improved the quality of this work and illuminated possible avenues for further work. ALQ acknowledges receipt of an STFC postgraduate studentship. NJW acknowledges receipt of an STFC Ernest Rutherford Fellowship (ref. ST/M005569/1) and a Leverhulme Trust Research Project Grant (RPG-2019-379). The authors would like to thank Janet Drew and Maria Monguió for discussions on Galactic rotation and its kinematic signature, and for bringing to our attention the work of Chevance et al. (2020) in the context of the large-scale proper motion signature seen in Cygnus that they had already seen in Carina at that time.

This paper makes uses of data processed by the Gaia Data Processing and Analysis Consortium (DPAC, https://www.cosmos.esa.int/w eb/gaia/dpac/consortium) and obtained by the Gaia mission from the European Space Agency (ESA) (https://www.cosmos.esa.int/gaia), as well as the INT Galactic Plane Survey (IGAPS) from the Isaac Newton Telescope (INT) operated in the Spanish Observatorio del Roque de los Muchachos. Data were also based on the Two-Micron All Star Survey, which is a combined mission of the Infrared Processing and Analysis Center/California Institute of Technology and the University of Massachusetts, along with The UKIDSS Galactic Plane Survey (GPS), a survey carried out by the UKIDSS consortium with the Wide Field Camera performing on the United Kingdom Infrared Telescope.

This work also benefited from the use of TOPCAT (Taylor 2017), ASTROPY (Astropy Collaboration 2013) and the Vizier and SIMBAD database, both operated at CDS, Strasbourg, France.

\section{DATA AVAILABILITY}

The data underlying this article will be uploaded to Vizier and will be available to access from there.

\section{REFERENCES}

Ambartsumian V. A., 1947, The Evolution of Stars and Astrophysics. Armenian SSR Academy of Sciences Press

Armstrong J. J., Wright N. J., Jeffries R. D., Jackson R. J., 2020, MNRAS, 494, 4794

Arnold B., Goodwin S. P., Wright N. J., 2020, MNRAS, 495, 3474

Astropy Collaboration, 2013, A\&A, 558, A33

Bailer-Jones C. A. L., 2015, Publ. Astron. Soc. Pac., 127, 994

Bailer-Jones C. A. L., Rybizki J., Fouesneau M., Demleitner M., Andrae R., 2020, ApJ, 161, 24

Barentsen G. et al., 2014, MNRAS, 444, 3230

Berlanas S. R., Wright N. J., Herrero A., Drew J. E., Lennon D. J., 2019, MNRAS, 484, 1838

Berlanas S. R. et al., 2020, A\&A, 642, A168

Blaauw A., 1964, ARA\&A, 2, 213

Blaha C., Humphreys R. M., 1989, AJ, 98, 1598

Bland-Hawthorn J., Gerhard O., 2016, ARA\&A, 54, 529

Bohlin R. C., 2007, in Sterken C., ed., ASP Conf. Ser. Vol. 364, HST Stellar Standards with 1 per cent Accuracy in Absolute Flux. Astron. Soc. Pac., San Francisco, p. 2269

Cantat-Gaudin T. et al., 2019, A\&A, 626, A17

Casey A. R., 2016, ApJS, 223, 8

Chevance M. et al., 2020, MNRAS, 493, 2872

Coelho P. R. T., 2014, MNRAS, 440, 1027

Comerón F., Pasquali A., 2012, A\&A, 543, A101

Cutri R. M. et al., 2003, 2MASS All Sky Catalog of Point Sources. NASA/IPAC Infrared Science Archive

Dobbs C. L., Pringle J. E., 2013, MNRAS, 432, 653

Dove J. B., Shull J. M., 1994, ApJ, 430, 222

Drew J.. E., Monguió M., Wright N., 2021, preprint (arXiv:2110.02081)

Drew J. E. et al., 2005, MNRAS, 362, 753

Drew J. E. et al., 2014, MNRAS, 440, 2036

Eilers A.-C., Hogg D. W., Rix H.-W., Ness M. K., 2019, ApJ, 871, 120

Ekström S. et al., 2012, A\&A, 537, A146

El-Badry K., Rix H.-W., Heintz T. M., 2021, MNRAS, 506, 2269

Fabricius C. et al., 2021, A\&A, 649, A5

Fitzpatrick E. L., Massa D., Gordon K. D., Bohlin R., Clayton G. C., 2019, ApJ, 886, 108

Foreman-Mackey D., Hogg D. W., Lang D., Goodman J., 2013, Publ. Astron. Soc. Pac., 125, 306

Gaia Collaboration, 2016, A\&A, 595, A1

Gaia Collaboration, 2021, A\&A, 649, A1

Garmany C. D., 1994, Publ. Astron. Soc. Pac., 106, 25

Green G. M., Schlafly E., Zucker C., Speagle J. S., Finkbeiner D., 2019, ApJ, 887, 93

Hills J. G., 1980, ApJ, 235, 986

Hodgkin S. T., Irwin M. J., Hewett P. C., Warren S. J., 2009, MNRAS, 394, 675

Humphreys R. M., McElroy D. B., 1984, ApJ, 284, 565

Ivezić Ž., Connelly A. J., VanderPlas J. T., Gray A., 2014, Statistics, Data Mining, and Machine Learning in Astronomy. Princeton University Press, Princeton University, New Jersey

Jordi C. et al., 2010, A\&A, 523, A48

Kiminki D. C. et al., 2007, ApJ, 664, 1102

Kounkel M. et al., 2018, AJ, 156, 84

Kruijssen J. M. D., 2012, MNRAS, 426, 3008

Lada C. J., Lada E. A., 2003, ARA\&A, 41, 57

Lindegren L. et al., 2020a, A\&A, 649, 31

Lindegren L. et al., 2020b, A\&A, 649, 35

Lucas P. W. et al., 2008, MNRAS, 391, 136

Maíz Apellániz J., Pantaleoni González M., Barbá R. H., 2021, A\&A, 649, A13

Martins F., Schaerer D., Hillier D. J., 2005, A\&A, 436, 1049

Maschberger T., 2013, MNRAS, 429, 1725

Massey P., Thompson A. B., 1991, AJ, 101, 1408

McKee C. F., Williams J. P., 1997, ApJ, 476, 144

Mel'nik A. M., Dambis A. K., 2017, MNRAS, 472, 3887 
Mel'Nik A. M., Efremov Y. N., 1995, Astron. Lett., 21, 10

Melnik A. M., Dambis A. K., 2020, MNRAS, 493, 2339

Monguió M. et al., 2020, A\&A, 638, A18

Orellana R. B., De Biasi M. S., Paíz L. G., 2021, MNRAS, 502, 6080

Pecaut M. J., Mamajek E. E., 2013, ApJS, 208, 9

Pedregosa F. et al., 2011, J. Mach. Learn. Res., 12, 2825

Rauch T., Deetjen J. L., 2003, in Hubeny I., Mihalas D., Werner K., eds, ASP Conf. Proc. Vol. 288, Stellar Atmosphere Modeling. Astron. Soc. Pac., San Francisco, p. 103

Reipurth B., Schneider N., 2008, Star Formation and Young Clusters in Cygnus. The Northern Sky ASP Monograph Publications, University of Hawaii, p. 36

Riello M. et al., 2020, A\&A, 649, A3

Schönrich R., Binney J., Dehnen W., 2010, MNRAS, 403, 1829

Shull J. M., Saken J. M., 1995, ApJ, 444, 663

Skrutskie M. F. et al., 2006, AJ, 131, 1163

Smith M., 2016, PhD thesis, University of Hertfordshire

Straižys V., Lazauskaitė R., 2009, Balt. Astron., 18, 19

Taylor M., 2005, in Shopbell P., Britton M., Ebert R., eds, ASP Conf. Ser. Vol. 347, Astronomical Data Analysis Software and Systems XIV. Astron. Soc. Pac., San Francisco, p. 29

Trundle C., Dufton P. L., Hunter I., Evans C. J., Lennon D. J., Smartt S. J., Ryans R. S. I., 2007, A\&A, 471, 625
Verbeek K. et al., 2012, MNRAS, 420, 1115

Ward J. L., Kruijssen J. M. D., 2018, MNRAS, 475, 5659

Werner K., Dreizler S., 1999, J. Computat. Appl. Math., 109, 65

Werner K., Deetjen J. L., Dreizler S., Nagel T., Rauch T., Schuh S. L., 2003, in Hubeny I., Mihalas D., Werner K., eds, ASP Conf. Ser. Vol. 288, Stellar Atmosphere Modeling. Astron. Soc. Pac., San Francisco, p. 31

Wright N. J., 2020, New Astron. Rev., 90, 101549

Wright N. J., Mamajek E. E., 2018, MNRAS, 476, 381

Wright N. J., Drew J. E., Mohr-Smith M., 2015, MNRAS, 449, 741

Wright N. J., Bouy H., Drew J. E., Sarro L. M., Bertin E., Cuillandre J.-C., Barrado D., 2016, MNRAS, 460, 2593

Zari E., Rix H. W., Frankel N., Xiang M., Poggio E., Drimmel R., Tkachenko A., 2021, A\&A, 650, A112

\section{APPENDIX A: COMPARISON BETWEEN OUR GROUPS}

Figs A1 and A2 show a visual comparison between our new groups and, respectively, the historical $\mathrm{OB}$ associations in this part of Cygnus and the groups identified using DBSCAN. See Sections 4.3 and 4.4 for a discussion of the overlap in membership between our groups and these groups.
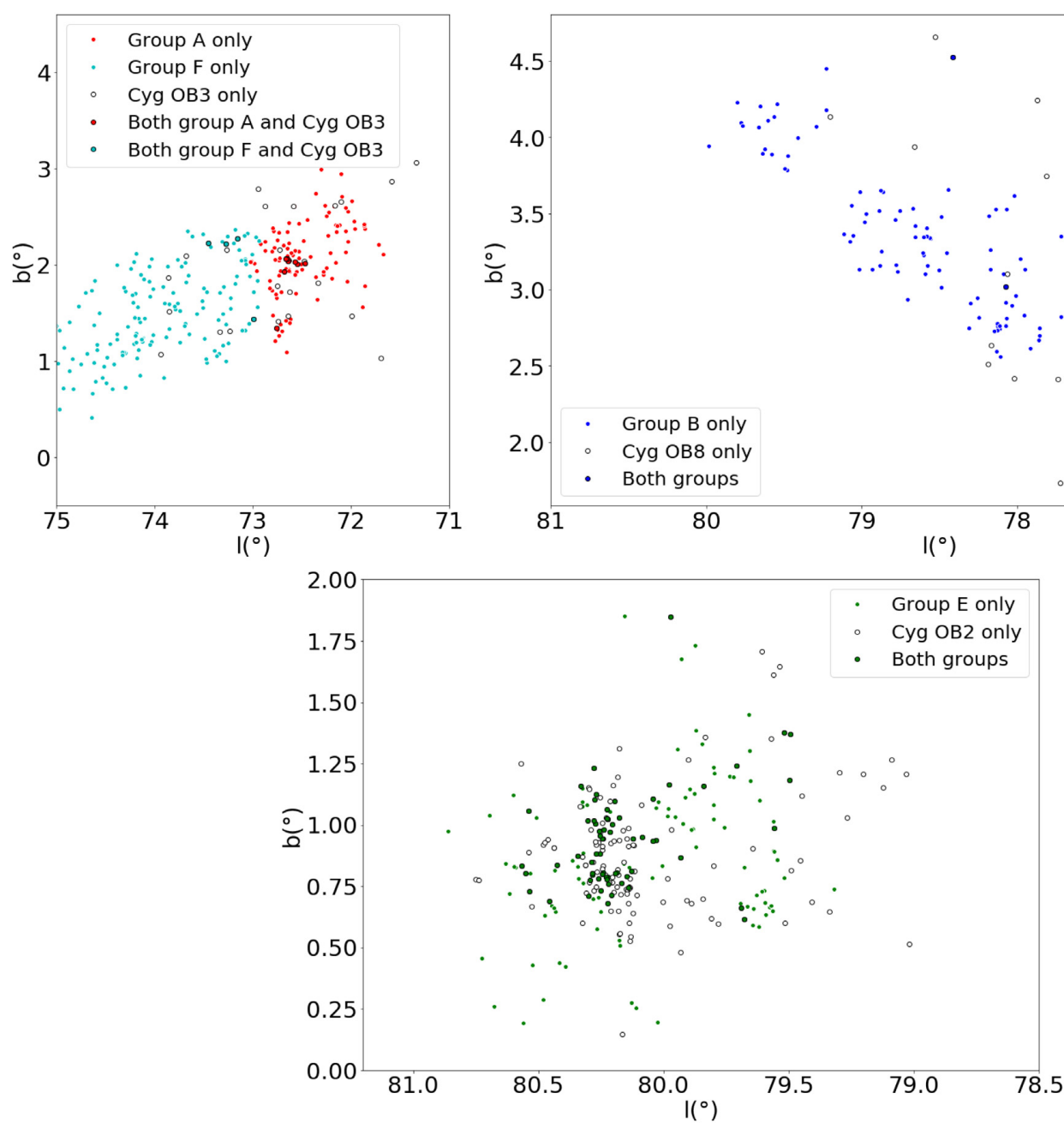

Figure A1. Comparison between our new groups and the historical associations Cyg OB2, OB3, and OB8. 

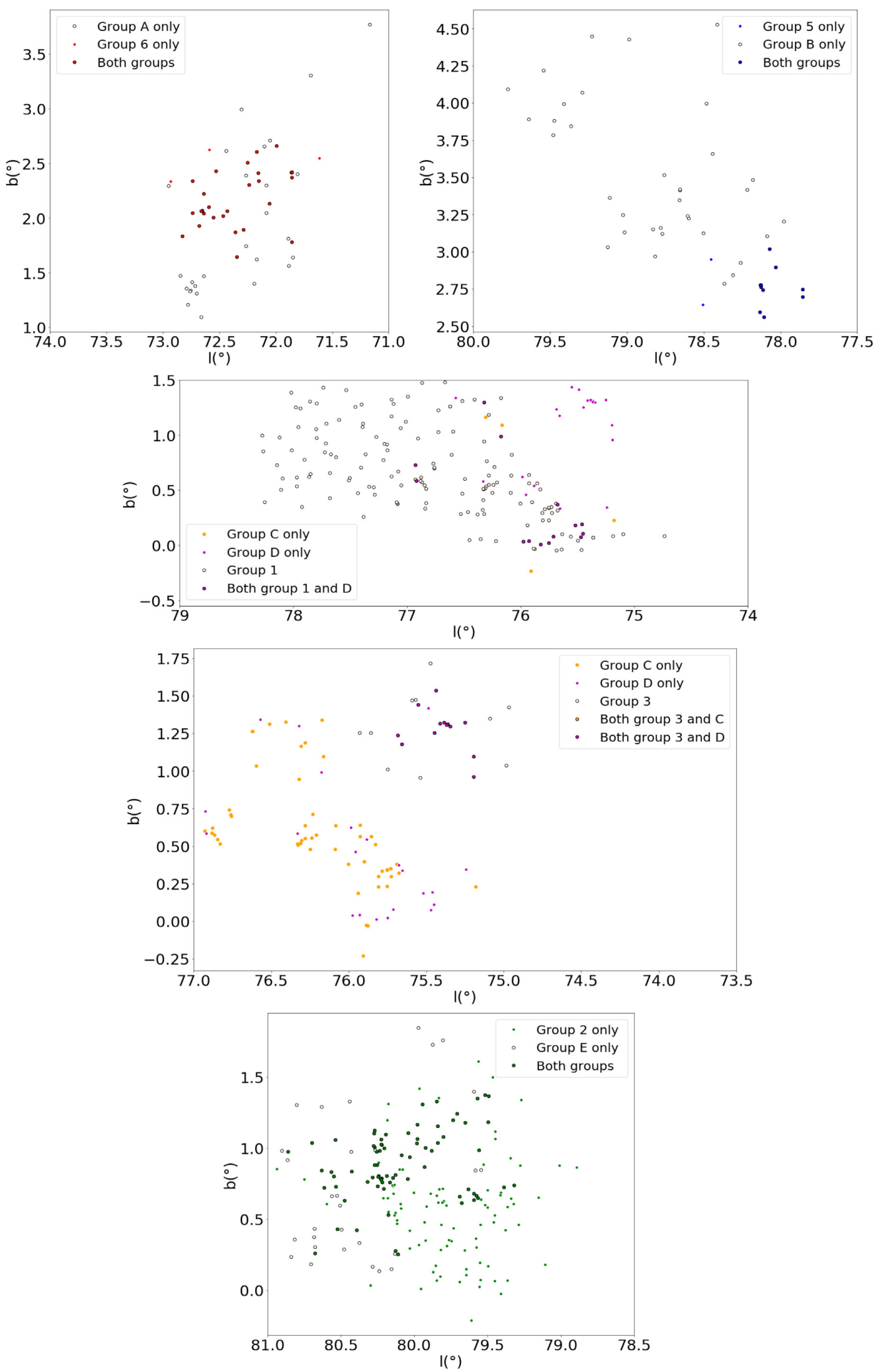

Figure A2. Comparison between our new groups and the groups identified by DBSCAN.

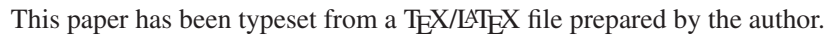

